\title{
MONOTONE DIFFERENCE SCHEMES STABILIZED BY DISCRETE MOLLIFICATION FOR STRONGLY DEGENERATE PARABOLIC EQUATIONS
}

\author{
CARLOS D. ACOSTA ${ }^{\mathrm{A}}$, RAIMUND BÜRGER ${ }^{\mathrm{B}}$, AND CARLOS E. MEJÍA ${ }^{\mathrm{C}}$
}

\begin{abstract}
The discrete mollification method is a convolution-based filtering procedure suitable for the regularization of ill-posed problems and for the stabilization of explicit schemes for the solution of PDEs. This method is applied to the discretization of the diffusive terms of a known first-order monotone finite difference scheme [S. Evje and K.H. Karlsen, SIAM J Numer Anal 37 (2000) 1838-1860] for initial value problems of strongly degenerate parabolic equations in one space dimension. It is proved that the mollified scheme is monotone, and converges to the unique entropy solution of the initial value problem, under a CFL stability condition which permits to use time steps that are larger than with the un-mollified (basic) scheme. Several numerical experiments illustrate the performance, and gains in CPU time, for the mollified scheme. Applications to initial-boundary value problems are included.
\end{abstract}

\section{INTRODUCTION}

1.1. Scope. This paper is concerned with finite difference methods for the following initial value problem for a degenerate parabolic equation:

$$
\begin{aligned}
u_{t}+f(u)_{x} & =A(u)_{x x}, \quad(x, t) \in \Pi_{T}:=\mathbb{R} \times(0, T), \quad T>0, \\
u(x, 0) & =u_{0}(x), \quad x \in \mathbb{R},
\end{aligned}
$$

where the integrated diffusion coefficient $A$ is defined by

$$
A(u)=\int_{0}^{u} a(s) \mathrm{d} s, \quad a(u) \geq 0, \quad a \in L^{\infty}(\mathbb{R}) \cap L^{1}(\mathbb{R}) .
$$

The function $a$ is allowed to vanish on $u$-intervals of positive length, on which (1.1) degenerates to a firstorder scalar conservation law. Therefore, (1.1) is called strongly degenerate. It is well known that solutions of (1.1), (1.2) are, in general, discontinuous even if $u_{0}$ is smooth, and need to be defined as weak solutions along with an entropy condition to select the physically relevant solution, the entropy solution. Applications of degenerate parabolic equations include two-phase flow in porous media, traffic flow, and sedimentationconsolidation processes.

Evje and Karlsen [1] introduced an explicit monotone difference scheme for the approximation of entropy solutions of (1.1), (1.2) based on the first-order accurate, monotone Engquist-Osher numerical flux [2] for the convective part combined with a conservative discretization of the degenerate diffusion term. If $\Delta x$ and $\Delta t$ denote the spatial meshwidth and the time step, respectively, they proved convergence of the scheme to an entropy solution as $\Delta x, \Delta t \downarrow 0$ provided that the following CFL stability condition is satisfied:

$$
\lambda\left\|f^{\prime}\right\|_{\infty}+2 \mu\|a\|_{\infty} \leq 1, \quad \lambda:=\Delta t / \Delta x, \quad \mu:=\Delta t / \Delta x^{2} .
$$

Similar conditions appear for explicit finite difference schemes approximating smooth solutions of strictly parabolic convection-diffusion equations. For these equations, the so-called method of discrete mollification $[3,4]$ consists in using certain convex combinations of finite difference stencils rather than a single one. This

\footnotetext{
Date: December 3, 2009.

A Universidad Nacional de Colombia, Department of Mathematics and Statistics, Manizales, Colombia. E-Mail: cdacostam@unal.edu.co.

${ }^{\mathrm{B}} \mathrm{CI}^{2} \mathrm{MA}$ and Departamento de Ingeniería Matemática, Facultad de Ciencias Físicas y Matemáticas, Universidad de Concepción, Casilla 160-C, Concepción, Chile. E-Mail: rburger@ing-mat.udec.cl.

${ }^{\mathrm{C}}$ Universidad Nacional de Colombia, Department of Mathematics, Medellín, Colombia. E-Mail: cemejia@unal.edu.co.
} 
device leads to a consistent numerical method with a new CFL condition that allows to use larger time steps, i.e. it stabilizes the given method.

In our case, the application of discrete mollification to the scheme introduced in [1] leads to a mollified scheme whose CFL stability condition is given by

$$
\lambda\left\|f^{\prime}\right\|_{\infty}+2 \mu \varepsilon_{\eta}\|a\|_{\infty} \leq 1, \quad \text { where } \varepsilon_{\eta}:=C_{\eta}\left(1-w_{0}\right) \in(0,1),
$$

where $C_{\eta}$ and $w_{0}$ are a parameter and the central weight, respectively, of the discrete mollification operator that depend on the width $\eta$ of the mollification stencil. Since $\varepsilon_{\eta} \in(0,1)$, for given $\Delta x$ the condition (1.5) admits to employ values of $\Delta t$ that are up to several times larger than for the standard, un-mollified version of the scheme with the restriction (1.4). This accelerates the given scheme but, as our numerical experiments show, introduces an at most moderate additional error.

It is the purpose of this paper to demonstrate that discrete mollification can also be applied to difference methods for the initial value problem for a strongly degenerate parabolic equation (1.1), (1.2). We prove that the new method is monotone and converges to the unique entropy solution of (1.1), (1.2) under the less restrictive CFL condition (1.5). The performance of the method is illustrated in several numerical examples.

1.2. Related work. Discrete mollification $[5,6]$ is a versatile convolution-based filtering procedure for the regularization of ill-posed problems $[6,7,8,9]$ and the stabilization of explicit schemes for the solution of PDEs. In [3] and [4] the mollification method was introduced as a stabilizer for numerical schemes for strictly parabolic convection-diffusion equations and non-linear scalar conservation laws, respectively. In [10] it is shown that a particular discrete approximation of the second derivative of a smooth function, based on discrete mollification, stabilizes operator splitting methods [11] for the numerical solution of convectiondiffusion problems. The method of [10] is applied herein to strongly degenerate parabolic equations.

On the other hand, monotone schemes for first-order conservation laws (corresponding to $A \equiv 0$ ) were first analyzed in $[12,13]$. Their attractive feature is the convergence to an entropy solution, which remains valid for the application to strongly degenerate parabolic equations. This was first exploited by Evje and Karlsen in [1]. Related analyses include implicit monotone schemes for degenerate parabolic equations [14], problems with boundary conditions [15], multidimensional degenerate parabolic equations [16], equations with discontinuous coefficients [17, 18, 19], and problems of parameter identification [20] (this list is far from being complete). Of course, the robustness of monotone schemes, in particular the convergence to the entropy solution, comes at the well-known price of the generic limitation to first-order accuracy.

1.3. Outline of the paper. The remainder of the paper is organized as follows. In Section 2 we present preliminary material, including a definition of an entropy solution of (1.1), (1.2) in Section 2.1, a description of the unmollified (basic) scheme from [1] in Section 2.2, a precise statement of the assumptions underlying the convergence analysis (Section 2.3), and an outline of the discrete mollification operator and its properties (Section 2.4). Section 3 deals with the mollified scheme, which is motivated in Section 3.1, and whose convergence to an entopy solution of (1.1), (1.2) is shown in Section 3.2. Based on standard compactness arguments, we prove that under the CFL condition (1.5) the mollified scheme is conservative and monotone, and produces uniformly bounded approximate solutions that satisfy the $L^{1}$ Lipschitz continuity in time property. Moreover, under an additional limitation of the choice of the mollification stencil the approximations of the integrated diffusion coefficient have the appropriate regularity properties. Since the scheme is monotone, it satisfies a discrete entropy inequality, and by a Lax-Wendroff-type argument we prove that it converges to an entropy solution of (1.1), (1.2) as $\Delta t, \Delta x \downarrow 0$. The mollified scheme is further supported by numerical experiments presented in Section 4, which are motivated by three different applicative models that also include boundary conditions. Some conclusions are collected in Section 5.

\section{Preliminaries}

2.1. Definition of an entropy solution. We recall here the definition of entropy solutions of (1.1), (1.2) from [1].

Definition 2.1. A bounded measurable function $u$ is said to be an entropy solution of (1.1), (1.2) if it satisfies 
(1) $u \in B V\left(\Pi_{T}\right)$ and $A(u) \in C^{1,1 / 2}\left(\bar{\Pi}_{T}\right)$.

(2) For all non-negative test functions $\phi \in C_{0}^{\infty}\left(\Pi_{T}\right)$ with $\left.\phi\right|_{t=T}=0$ and any $c \in \mathbb{R}$, the following entropy Kružkov-type inequality is satisfied:

$$
\begin{aligned}
& \iint_{\Pi_{T}}\left\{|u-c| \phi_{t}+\operatorname{sgn}(u-c)(f(u)-f(c)) \phi_{x}+|A(u)-A(c)| \phi_{x x}\right\} \mathrm{d} t \mathrm{~d} x \\
& \quad+\int_{\mathbb{R}}\left|u_{0}-c\right| \phi(x, 0) \mathrm{d} x \geq 0 .
\end{aligned}
$$

It is well known that entropy solutions of $(1.1),(1.2)$ in the sense of Definition 2.1 are $L^{1}$-contractive, i.e., if $u^{1}$ and $u^{2}$ are two entropy solutions corresponding to the respective initial data $u_{0}^{1}$ and $u_{0}^{2}$, then $\left\|u^{1}(\cdot, t)-u^{2}(\cdot, t)\right\|_{1} \leq\left\|u_{0}^{1}-u_{0}^{2}\right\|_{1}$ for $0 \leq t \leq T$. In particular, entropy solutions of (1.1), (1.2) are unique. This is, in fact, valid for initial value problems of more general strongly degenerate parabolic equations, also in several space dimensions. See [21] for details.

2.2. The unmollified scheme (basic scheme). We select a mesh size $\Delta x>0$ and a time step $\Delta t>0$ such that there exists an integer $N$ with $N \Delta t=T$. Let $u_{j}^{n}$ denote the value of the difference approximation at $(j \Delta x, n \Delta t)$ for $j \in \mathbb{Z}$ and $n=1, \ldots, N$. We then discretize the initial datum by

$$
u_{j}^{0}:=\frac{1}{\Delta x} \int_{(j-1 / 2) \Delta x}^{(j+1 / 2) \Delta x} u_{0}(x) \mathrm{d} x, \quad j \in \mathbb{Z},
$$

and calculate the solution values at time level $t^{n+1}$ from those at time $t^{n}$ by the explicit marching formula

$$
u_{j}^{n+1}=u_{j}^{n}-\lambda \Delta_{+} F^{\mathrm{EO}}\left(u_{j-1}^{n}, u_{j}^{n}\right)+\mu \Delta^{2} A\left(u_{j}^{n}\right), \quad j \in \mathbb{Z}, \quad n=0,1,2, \ldots, N-1,
$$

where we define the standard difference operators

$$
\Delta_{+} V_{j-1}=\Delta_{-} V_{j}=\Delta V_{j-1 / 2}:=V_{j}-V_{j-1}, \quad \Delta_{0} V_{j}:=\left(V_{j+1}-V_{j-1}\right) / 2, \quad \Delta^{2} V_{j}:=V_{j+1}-2 V_{j}+V_{j-1},
$$

and use the Engquist-Osher [2] numerical flux given by $F^{\mathrm{EO}}\left(u_{j}^{n}, u_{j+1}^{n}\right)=f^{+}\left(u_{j}^{n}\right)+f^{-}\left(u_{j+1}^{n}\right)$, where

$$
f^{+}(u):=f(0)+\int_{0}^{u} \max \left\{f^{\prime}(s), 0\right\} \mathrm{d} s, \quad f^{-}(u):=\int_{0}^{u} \min \left\{f^{\prime}(s), 0\right\} \mathrm{d} s .
$$

Under the CFL condition (1.4) the scheme (2.3) is monotone, therefore first-order accurate, and converges to the unique entropy solution [1]. The new scheme will be based on replacing the term $\mu \Delta^{2} A\left(u_{j}^{n}\right)$ in (2.3) by a different expression involving discrete mollification. We will therefore refer to (2.3) as the basic scheme.

2.3. Assumptions. With the notation related to the discretization at hand, we state, similarly to [15], as a further assumption that

$$
u_{0} \in \mathcal{B}:=\left\{u \in B V(\mathbb{R}) \mid \exists M_{1}, M_{2}>0: u_{0}(x)=\text { const. for } x \notin\left[-M_{1}, M_{1}\right], \quad \operatorname{TV}\left(A\left(u_{0}\right)_{x}\right) \leq M_{2}\right\} .
$$

This means, in particular, that there exists a constant $M_{3}$ such that

$$
\sum_{m \in \mathbb{Z}}\left|\Delta^{2} A\left(u_{m}^{0}\right)\right| \leq M_{3} \Delta x \quad \text { uniformly in } \Delta x .
$$

2.4. The discrete mollification operator. The mollification method $[5,6]$ is based on replacing the discrete function $y=\left\{y_{j}\right\}_{j \in \mathbb{Z}}$, which can, for example, consist of evaluations or cell averages of a real function $y=y(x)$ given at equidistant grid points $x_{j}=x_{0}+j \Delta x, \Delta x>0, j \in \mathbb{Z}$, by its mollified version $J_{\eta} y$, where $J_{\eta}$ is the so-called mollification operator defined by

$$
\left[J_{\eta} y\right]_{j}:=\sum_{i=-\eta}^{\eta} w_{i} y_{j-i}, \quad j \in \mathbb{Z},
$$




\begin{tabular}{|c|c|c|c|c|c|c|c|c|c|c|}
\hline$\eta$ & $i=0$ & $i=1$ & $i=2$ & $i=3$ & $i=4$ & $i=5$ & $i=6$ & $i=7$ & $i=8$ & $\zeta$ \\
\hline 0 & 1 & & & & & & & & & - \\
\hline 1 & 0.84272 & 0.07864 & & & & & & & & 0.0786 \\
\hline 2 & 0.60387 & 0.19262 & $5.4438 \mathrm{e}-3$ & & & & & & & 0.1926 \\
\hline 3 & 0.45556 & 0.23772 & $3.3291 \mathrm{e}-2$ & $1.2099 \mathrm{e}-3$ & & & & & & 0.2341 \\
\hline 4 & 0.36266 & 0.24003 & $6.9440 \mathrm{e}-2$ & $8.7275 \mathrm{e}-3$ & $4.7268 \mathrm{e}-4$ & & & & & 0.2101 \\
\hline 5 & 0.30028 & 0.22625 & $9.6723 \mathrm{e}-2$ & $2.3430 \mathrm{e}-2$ & $3.2095 \mathrm{e}-3$ & $2.4798 \mathrm{e}-4$ & & & & 0.1266 \\
\hline 6 & 0.25585 & 0.20831 & 0.11241 & $4.0192 \mathrm{e}-2$ & $9.5154 \mathrm{e}-3$ & $1.4905 \mathrm{e}-3$ & $1.5434 \mathrm{e}-4$ & & & -0.0145 \\
\hline 7 & 0.22270 & 0.19058 & 0.11942 & $5.4793 \mathrm{e}-2$ & $1.8403 \mathrm{e}-2$ & $4.5234 \mathrm{e}-3$ & $8.1342 \mathrm{e}-4$ & $1.0697 \mathrm{e}-4$ & & -0.2121 \\
\hline 8 & 0.19708 & 0.17444 & 0.12097 & $6.5725 \mathrm{e}-2$ & $2.7973 \mathrm{e}-2$ & $9.3255 \mathrm{e}-3$ & $2.4348 \mathrm{e}-3$ & $4.9782 \mathrm{e}-4$ & $7.9691 \mathrm{e}-5$ & -0.4660 \\
\hline
\end{tabular}

TABLE 1. Discrete mollification weights $w_{i}$ given by $(2.7)$ with $p=3$, along with the value of $\zeta$ defined in (3.15) (see Lemma 3.5).

where $\eta \in \mathbb{N}$ is the support parameter (indicating the width of the mollification stencil) and the so-called weights $w_{i}$ satisfy

$$
w_{i}=w_{-i}, \quad 0 \leq w_{i} \leq w_{i-1}, \quad i=1, \ldots, \eta ; \quad \sum_{i=-\eta}^{\eta} w_{i}=1 .
$$

The weights $w_{i}$ are obtained by numerical integration of the truncated Gaussian kernel

$$
\kappa_{p \delta}(t):=\left\{\begin{array}{ll}
A_{p} \delta^{-1} \exp \left(-t^{2} / \delta^{2}\right) & \text { for }|t| \leq p \delta, \\
0 & \text { otherwise, }
\end{array} \quad \text { where } A_{p}:=\left(\int_{-p}^{p} \exp \left(-s^{2}\right) \mathrm{d} s\right)^{-1},\right.
$$

and $\delta$ and $p$ are positive parameters. This kernel satisfies $\kappa_{p \delta} \geq 0, \kappa_{p \delta} \in C^{\infty}(-p \delta, p \delta), \kappa_{p \delta}=0$ outside $[-p \delta, p \delta]$, and $\int_{\mathbb{R}} \kappa_{p \delta}=1$. Then we define $\xi_{j-1 / 2}:=(j-1 / 2) \Delta x$ for $j \in \mathbb{Z}$ and compute the weights by

$$
w_{i}:=\int_{\xi_{i-1 / 2}}^{\xi_{i+1 / 2}} \kappa_{p \delta}(-s) \mathrm{d} s, \quad i=-\eta, \ldots, \eta
$$

Usually $p=3$ is taken and $\delta$, whose role is to determine the shape of the kernel's Gaussian bell, is considered as regularization parameter, and it is estimated by means of methods like Generalized Cross Validation (GCV) [6, 8]. In any case, in this work the main relationship between $\delta$ and $\eta$ is given by $\delta=(\eta+1 / 2) \Delta x / p$. This choice generates weights $w_{-\eta}, \ldots, w_{\eta}$, that are independent of $\Delta x$. The resulting values of $w_{i}$ for several values of $\eta$ and $p=3$ are given in Table 1.

We conclude this section with some approximation and stability results.

Lemma 2.1. The discrete mollification operator can be written in the forms

$$
\left[J_{\eta} y\right]_{j}=y_{j}+\left(\psi_{j}-\psi_{j-1}\right)=y_{j}-\sum_{i=1}^{\eta} \rho_{i} \Delta y_{j-i+1 / 2}+\sum_{i=1}^{\eta} \rho_{i} \Delta y_{j+i-1 / 2},
$$

where we define

$$
\begin{gathered}
\psi_{j}:=\sum_{k=1}^{\eta} \rho_{k}\left(y_{j+k}-y_{j-k+1}\right)=\sum_{k=-\eta+1}^{\eta-1} Q_{-k} \Delta y_{j+k+1 / 2}, \\
\rho_{k}:=\sum_{i=k}^{\eta} w_{i}, \quad k=-\eta, \ldots, \eta ; \quad Q_{-k}=Q_{k}:=\sum_{i=k+1}^{\eta} \rho_{i}, \quad k=0, \ldots, \eta-1 .
\end{gathered}
$$

We assume that $g$ is a sufficiently smooth real function, set $y_{j}=g\left(x_{j}\right)$, and employ the Taylor expansion

$$
y_{j+i}=y_{j}+(i \Delta x) g^{\prime}\left(x_{j}\right)+\frac{1}{2}(i \Delta x)^{2} g^{\prime \prime}\left(x_{j}\right)+\frac{1}{6}(i \Delta x)^{3} g^{\prime \prime \prime}\left(x_{j}\right)+\frac{1}{24}(i \Delta x)^{4} g^{(4)}\left(\xi_{j, i}\right),
$$


where $\xi_{j, i}$ is a real number between $x_{j}$ and $x_{j+i}$. Then, defining

$$
C_{\eta}:=\left(\sum_{i=-\eta}^{\eta} i^{2} w_{-i}\right)^{-1}
$$

we can write

$$
\left[J_{\eta} y\right]_{j}=\sum_{i=-\eta}^{\eta} w_{-i} y_{j+i}=y_{j}+\frac{\Delta x^{2}}{2 C_{\eta}} g^{\prime \prime}\left(x_{j}\right)+\frac{\Delta x^{4}}{24} \sum_{i=-\eta}^{\eta} i^{4} w_{-i} g^{(4)}\left(\xi_{j, i}\right) .
$$

Theorem 2.1. Let $g \in C^{4}(\mathbb{R})$ with $g^{(4)}$ bounded on $\mathbb{R}$, and set $y_{j}=g\left(x_{j}\right)$. If the data $\left\{y_{j}^{\varepsilon}\right\}_{j \in \mathbb{Z}}$ satisfy

$$
\left|y_{j}^{\varepsilon}-y_{j}\right| \leq \varepsilon \quad \text { for all } j \in \mathbb{Z}
$$

then

$$
\left|\left[J_{\eta} y^{\varepsilon}\right]_{j}-\left[J_{\eta} y\right]_{j}\right| \leq \varepsilon \quad \text { for all } j \in \mathbb{Z} .
$$

Additionally, for each compact set $K=[a, b]$ there exists a constant $C=C(K)$ such that

$$
\left|\left[J_{\eta} y\right]_{j}-g\left(x_{j}\right)-\frac{\Delta x^{2}}{2 C_{\eta}} g^{\prime \prime}\left(x_{j}\right)\right| \leq C \Delta x^{4} \quad \text { for all } j \in \mathbb{Z} .
$$

Moreover, the following inequalities hold for all $j \in \mathbb{Z}$, where $C$ is a different constant in each inequality:

$$
\begin{aligned}
\left|\left[J_{\eta} y\right]_{j}-g\left(x_{j}\right)\right| & \leq C \Delta x^{2}, & \left|\Delta_{0}\left[J_{\eta} y\right]_{j}-\Delta x g^{\prime}\left(x_{j}\right)\right| \leq C \Delta x^{3}, \\
\left|\Delta_{+}\left[J_{\eta} y\right]_{j}-\Delta x g^{\prime}\left(x_{j}\right)\right| & \leq C \Delta x^{2}, & \left|\Delta_{-} \Delta_{+}\left[J_{\eta} y\right]_{j}-\Delta x^{2} g^{\prime \prime}\left(x_{j}\right)\right| \leq C \Delta x^{4} .
\end{aligned}
$$

Details of the proofs of Lemma 2.1 and of the third part of Theorem 2.1 can be found in [3], while (2.11) is a way of rewriting $(2.10)$.

\section{Mollified SCHEME}

3.1. Motivation of the mollified scheme. The new scheme is based on the consistency result for discrete mollification (2.11), which implies the approximation

$$
g^{\prime \prime}\left(x_{j}\right)=\frac{2 C_{\eta}}{\Delta x^{2}}\left(\left[J_{\eta} g\right]_{j}-g\left(x_{j}\right)\right)+\mathcal{O}\left(\Delta x^{2}\right) \quad \text { as } \Delta x \downarrow 0 .
$$

Assume now for the moment that $A(u)$ is a smooth function of $x$. Then we have

$$
\begin{aligned}
\frac{1}{\Delta x^{2}} \Delta^{2} A\left(u\left(x_{j}\right)\right) & =\left.A(u)_{x x}\right|_{x=x_{j}}+\mathcal{O}\left(\Delta x^{2}\right), \\
\frac{2 C_{\eta}}{\Delta x^{2}}\left(\left[J_{\eta} A(u)\right]_{j}-A\left(u\left(x_{j}\right)\right)\right) & =\left.A(u)_{x x}\right|_{x=x_{j}}+\mathcal{O}\left(\Delta x^{2}\right),
\end{aligned}
$$

so we obtain the following new consistent scheme if we replace the expression $\mu \Delta^{2} A\left(u_{j}^{n}\right)$ in the right-hand side of $(2.3)$ by $2 \mu C_{\eta}\left(\left[J_{\eta} A(u)\right]_{j}-A\left(u_{j}^{n}\right)\right)$ :

$$
u_{j}^{n+1}=u_{j}^{n}-\lambda \Delta_{+} F^{\mathrm{EO}}\left(u_{j-1}^{n}, u_{j}^{n}\right)+2 \mu C_{\eta}\left(\left[J_{\eta} A\left(u^{n}\right)\right]_{j}-A\left(u_{j}^{n}\right)\right) .
$$

As we will see, the new scheme (3.2) has a more favorable CFL condition than (2.3). For strictly parabolic convection-diffusion problems where $A$ is indeed smooth, (3.2) represents an obviously consistent scheme (see [10]). For the present setting, where we wish to approximate discontinuous solutions, and $A$ is only Lipschitz continuous, this calculus only serves as a motivation for the new scheme. However, as we will show, in the present case the scheme is justified and supported by a convergence analysis, since the Lax-Wendroff-type argument invoked to show that the scheme converges to an entropy solution will appeal to (3.1) only with $g$ replaced by a smooth test function. 


\subsection{Convergence analysis of the mollified scheme.}

Lemma 3.1. The scheme (3.2) is conservative, and its numerical flux is consistent with (1.1).

Proof. According to Lemma 2.1 the mollification operator is indeed conservative and we can write

$$
\left[J_{\eta} A\left(u^{n}\right)\right]_{j}=A\left(u_{j}^{n}\right)+\psi_{j}^{n}-\psi_{j-1}^{n},
$$

where we define

$$
\psi_{j}^{n}:=\sum_{k=1}^{\eta} \rho_{k}\left(A\left(u_{j+k}^{n}\right)-A\left(u_{j-k+1}^{n}\right)\right), \quad \text { where } \rho_{k}=\sum_{i=k}^{\eta} w_{i},
$$

so the marching formula (3.2) can be rewritten as

$$
u_{j}^{n+1}=u_{j}^{n}-\lambda \Delta_{+} F^{\mathrm{EO}}\left(u_{j-1}^{n}, u_{j}^{n}\right)+2 \mu C_{\eta} \Delta_{+} \psi_{j-1}^{n} .
$$

The consistency follows by recalling (3.1), and by noting that $F^{\mathrm{EO}}(u, u)=f(u)$ and $\psi_{j}^{n}=0$ if $u_{k}=u$ for $k=j-\eta, \ldots, j+\eta$.

For later purposes, we remark that due to Lemma 2.1, we can write

$$
\psi_{j}^{n}=\sum_{k=-\eta+1}^{\eta-1} Q_{k}\left(A\left(u_{j+k+1}^{n}\right)-A\left(u_{j+k}^{n}\right)\right)
$$

where the quantities $Q_{0}, \ldots, Q_{\eta-1}$ are defined in (2.9).

Lemma 3.2. The scheme (3.2) is monotone under the CFL condition (1.5), and we have the following uniform $L^{\infty}$ bound:

$$
\left\|u^{n}\right\|_{\infty} \leq\left\|u_{0}\right\|_{\infty} \quad \text { for all } n=1, \ldots, N
$$

Proof. We denote by $u^{n}$ and $v^{n}$ the data $\left\{u_{i}^{n}\right\}_{i \in \mathbb{Z}}$ and $\left\{u_{i}^{n}\right\}_{i \in \mathbb{Z}}$, respectively, and assume that $v_{i}^{n}=u_{i}^{n}$ for $i \in \mathbb{Z}$ with the exception of $i=k$, for which we assume that $u_{k}^{n} \leq v_{k}^{n}$. We write the scheme (3.2) as $u_{j}^{n+1}=\mathcal{S}_{j}\left(u^{n}\right)$, where $\mathcal{S}_{j}\left(u^{n}\right)$ denotes the right-hand side of (3.2).

First, we consider the case $j-\eta \leq k \leq j-2$, in which we simply get

$$
\mathcal{S}_{j}\left(u^{n}\right)-\mathcal{S}_{j}\left(v^{n}\right)=2 \mu C_{\eta}\left[J_{\eta}\left(A\left(u^{n}\right)-A\left(v^{n}\right)\right)\right]_{j}=2 \mu C_{\eta} \sum_{i=-\eta}^{\eta} w_{-i}\left(A\left(u_{j+i}^{n}\right)-A\left(v_{j+i}^{n}\right)\right) \leq 0,
$$

since $A$ is non-decreasing. In the case $k=j-1$ we get

$$
\mathcal{S}_{j}\left(u^{n}\right)-\mathcal{S}_{j}\left(v^{n}\right)=\lambda\left(F^{\mathrm{EO}}\left(u_{j-1}^{n}, u_{j}^{n}\right)-F^{\mathrm{EO}}\left(v_{j-1}^{n}, u_{j}^{n}\right)\right)+2 \mu C_{\eta}\left[J_{\eta}\left(A\left(u^{n}\right)-A\left(v^{n}\right)\right)\right]_{j} \leq 0,
$$

where we use that $F^{\mathrm{EO}}$ is non-decreasing in its first argument. The cases $k=j+1$ and $j+2 \leq k \leq j+\eta$ can be handled by similar arguments. The case $k=j$ requires special attention. We have

$$
\begin{aligned}
\mathcal{S}_{j}\left(u^{n}\right)-\mathcal{S}_{j}\left(v^{n}\right)= & u_{j}^{n}-v_{j}^{n}-\lambda\left(F^{\mathrm{EO}}\left(u_{j}^{n}, u_{j+1}^{n}\right)-F^{\mathrm{EO}}\left(v_{j}^{n}, u_{j+1}^{n}\right)\right)+\lambda\left(F^{\mathrm{EO}}\left(u_{j-1}^{n}, u_{j}^{n}\right)-F^{\mathrm{EO}}\left(u_{j-1}^{n}, v_{j}^{n}\right)\right) \\
& +2 \mu C_{\eta}\left[J_{\eta}\left(A\left(u^{n}\right)-A\left(v^{n}\right)\right)\right]_{j}-2 \mu C_{\eta}\left(A\left(u_{j}^{n}\right)-A\left(v_{j}^{n}\right)\right) .
\end{aligned}
$$

Considering that

$$
\left[J_{\eta}\left(A\left(u^{n}\right)-A\left(v^{n}\right)\right)\right]_{j}=\sum_{i=-\eta}^{\eta} w_{-i}\left(A\left(u_{j+i}^{n}\right)-A\left(v_{j+i}^{n}\right)\right)=w_{0}\left(A\left(u_{j}^{n}\right)-A\left(v_{j}^{n}\right)\right)
$$

and using that by the definition of $F^{\mathrm{EO}}$ we have

$$
\begin{aligned}
& F^{\mathrm{EO}}\left(u_{j}^{n}, u_{j+1}^{n}\right)-F^{\mathrm{EO}}\left(v_{j}^{n}, u_{j+1}^{n}\right)=f^{+}\left(u_{j}^{n}\right)-f^{+}\left(v_{j}^{n}\right), \\
& F^{\mathrm{EO}}\left(u_{j-1}^{n}, u_{j}^{n}\right)-F^{\mathrm{EO}}\left(u_{j-1}^{n}, v_{j}^{n}\right)=f^{-}\left(u_{j}^{n}\right)-f^{-}\left(v_{j}^{n}\right),
\end{aligned}
$$

we obtain

$$
\mathcal{S}_{j}\left(u^{n}\right)-\mathcal{S}_{j}\left(v^{n}\right)=u_{j}^{n}-v_{j}^{n}-\lambda\left(f^{+}\left(u_{j}^{n}\right)-f^{+}\left(v_{j}^{n}\right)\right)+\lambda\left(f^{-}\left(u_{j}^{n}\right)-f^{-}\left(v_{j}^{n}\right)\right)
$$




\begin{tabular}{llllllll}
\hline$\eta$ & 3 & 5 & 8 & 20 & 30 & 40 & 50 \\
\hline$\varepsilon_{\eta}$ & 0.71305 & 0.39686 & 0.19605 & 0.03917 & 0.01825 & 0.01051 & $6.82 \mathrm{e}-3$ \\
\hline
\end{tabular}

TABLE 2. Values of the coefficient $\varepsilon_{\eta}$ in the CFL condition (1.5) for $p=3$ and different values of $\eta$.

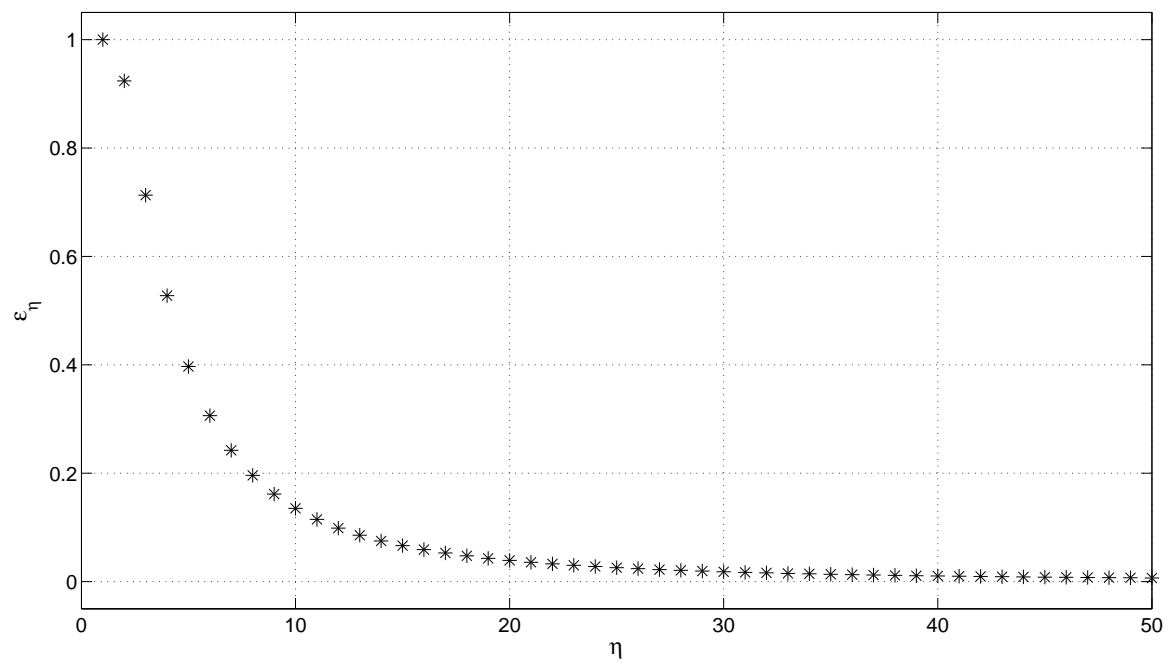

Figure 1. Values of the coefficient $\varepsilon_{\eta}$ in the CFL condition (1.5) for $p=3$ and different values of $\eta$.

$$
\begin{aligned}
& +2 \mu C_{\eta}\left(w_{0}-1\right)\left(A\left(u_{j}^{n}\right)-A\left(v_{j}^{n}\right)\right) \\
= & \int_{u_{k}^{n}}^{v_{k}^{n}}\left\{1-\lambda \max \left\{f^{\prime}(s), 0\right\}+\lambda \min \left\{f^{\prime}(s), 0\right\}-2 \mu C_{\eta}\left(1-w_{0}\right) a(s)\right\} \mathrm{d} s \\
\leq & -\int_{u_{k}^{n}}^{v_{k}^{n}}\left(1-\lambda\left|f^{\prime}(s)\right|-2 \mu C_{\eta}\left(1-w_{0}\right) a(s)\right) \mathrm{d} s .
\end{aligned}
$$

Under the condition (1.5) the integrand in (3.7) is non-negative, so $\mathcal{S}_{j}\left(u^{n}\right) \leq \mathcal{S}_{j}\left(v^{n}\right)$, and the scheme $(3.2)$ is monotone. The second assertion, (3.6), follows from the monotonicity by a standard argument if we take into account that if $w_{j}^{n}= \pm\left\|u_{0}\right\|_{\infty}$ for all $j \in \mathbb{Z}$ and $w_{j}^{n+1}=\mathcal{S}_{j}\left(w^{n}\right)$, then $w_{j}^{n+1}= \pm\left\|u_{0}\right\|_{\infty}$ for all $j \in \mathbb{Z}$; therefore, if $u^{n}$ are arbitrary data with $\left\|u^{n}\right\|_{\infty} \leq\left\|u_{0}\right\|_{\infty}$ and $u_{j}^{n+1}=\mathcal{S}_{j}\left(u^{n}\right)$, then $\left\|u^{n+1}\right\|_{\infty} \leq\left\|u_{0}\right\|_{\infty}$.

Remark 3.1. Since $0<w_{0} \leq 1$ and the constant $C_{\eta}$ defined in (3.1) satisfies $C_{\eta} \leq 1$ if $\eta$ is sufficiently large, the CFL condition (1.5) turns out to be less restrictive than (1.4). The actual value of $\varepsilon_{\eta}=C_{\eta}\left(1-w_{0}\right)$ for the usual choice $p=3$ and a range of $\eta$-values is shown in Table 2 and illustrated in Figure 1.

Since monotone schemes are total variation diminishing (TVD), we obtain the following corollary.

Corollary 3.1. Under the assumptions of Lemma 3.5 the numerical solution $\left\{u_{j}^{n}\right\}$ has the TVD property:

$$
\sum_{j \in \mathbb{Z}}\left|u_{j+1}^{n+1}-u_{j}^{n+1}\right| \leq \sum_{j \in \mathbb{Z}}\left|u_{j+1}^{n}-u_{j}^{n}\right| \quad \text { for all } n=0,1, \ldots, N-1 .
$$


Lemma 3.3. Assume that the CFL condition (1.5) is satisfied. Then there exists a constant $C_{1}$, which is independent of $\Delta t$ and $\Delta x$, such that

$$
\sum_{j \in \mathbb{Z}}\left|u_{j}^{n}-u_{j}^{m}\right| \leq C_{1} \lambda|n-m| \quad \text { for all } n, m \in\{0,1, \ldots, N\} .
$$

Proof. Let $n \in\{0,1, \ldots, N-1\}$. From (3.4) we obtain that

$$
u_{j}^{n+1}-u_{j}^{n}=u_{j}^{n}-u_{j}^{n-1}-\lambda \Delta_{-}\left(F^{\mathrm{EO}}\left(u_{j}^{n}, u_{j+1}^{n}\right)-F^{\mathrm{EO}}\left(u_{j}^{n-1}, u_{j+1}^{n-1}\right)\right)+2 \mu C_{\eta} \Delta_{-}\left(\psi_{j}^{n}-\psi_{j}^{n-1}\right) .
$$

Noting that

$$
F^{\mathrm{EO}}\left(u_{j}^{n}, u_{j+1}^{n}\right)-F^{\mathrm{EO}}\left(u_{j}^{n-1}, u_{j+1}^{n-1}\right)=D_{2, j}^{n}\left(u_{j+1}^{n}-u_{j+1}^{n-1}\right)+D_{1, j}^{n}\left(u_{j}^{n}-u_{j}^{n-1}\right),
$$

where $D_{1, j}^{n}=\partial_{1} F^{\mathrm{EO}}\left(v_{j}^{n-1 / 2}, u_{j+1}^{n-1}\right) \geq 0$ and $D_{2, j}^{n}=\partial_{2} F^{\mathrm{EO}}\left(u_{j}^{n}, \tilde{v}_{j+1}^{n-1 / 2}\right) \leq 0$ for some values $v_{j}^{n-1 / 2}, \tilde{v}_{j}^{n-1 / 2}$ between $u_{j}^{n}$ and $u_{j}^{n-1}$, we obtain

$$
\begin{aligned}
& \Delta_{-}\left(F^{\mathrm{EO}}\left(u_{j}^{n}, u_{j+1}^{n}\right)-F^{\mathrm{EO}}\left(u_{j}^{n-1}, u_{j+1}^{n-1}\right)\right) \\
& =D_{2, j}^{n}\left(u_{j+1}^{n}-u_{j+1}^{n-1}\right)-D_{1, j-1}^{n}\left(u_{j-1}^{n}-u_{j-1}^{n-1}\right)+\left(D_{1, j}^{n}-D_{2, j-1}^{n}\right)\left(u_{j}^{n}-u_{j}^{n-1}\right) .
\end{aligned}
$$

Moreover, we have that

$$
\begin{aligned}
\Delta_{-}\left(\psi_{j}^{n}-\psi_{j}^{n-1}\right) & =\left[J_{\eta} A\left(u^{n}\right)\right]_{j}-A\left(u_{j}^{n}\right)-\left(\left[J_{\eta} A\left(u^{n-1}\right)\right]_{j}-A\left(u_{j}^{n-1}\right)\right) \\
& =\sum_{k=-\eta}^{\eta} w_{-k}\left(A\left(u_{j+k}^{n}\right)-A\left(u_{j+k}^{n-1}\right)\right)-\left(A\left(u_{j}^{n}\right)-A\left(u_{j}^{n-1}\right)\right) \\
& =\sum_{k=-\eta}^{\eta} w_{-k} a\left(\bar{v}_{j+k}^{n-1 / 2}\right)\left(u_{j+k}^{n}-u_{j+k}^{n-1}\right)-a\left(\bar{v}_{j}^{n-1 / 2}\right)\left(u_{j}^{n}-u_{j}^{n-1}\right)
\end{aligned}
$$

for some $\bar{v}_{j+k-1 / 2}^{n}$ between $u_{j+k}^{n}$ and $u_{j+k-1}^{n}$. Consequently, we obtain

$$
\begin{aligned}
u_{j}^{n+1}-u_{j}^{n}= & \lambda D_{1, j-1}^{n}\left(u_{j-1}^{n}-u_{j-1}^{n-1}\right)-\lambda D_{2, j}^{n}\left(u_{j+1}^{n}-u_{j+1}^{n-1}\right) \\
& +\left(1-\lambda\left(D_{1, j}^{n}-D_{2, j-1}^{n}\right)-2 \mu C_{\eta} a\left(\bar{v}_{j}^{n-1 / 2}\right)\left(1-w_{0}\right)\right)\left(u_{j}^{n}-u_{j}^{n-1}\right) \\
& +2 \mu C_{\eta} \sum_{\substack{k=-\eta \\
k \neq 0}}^{\eta} w_{-k} a\left(\bar{v}_{j+k}^{n-1 / 2}\right)\left(u_{j+k}^{n}-u_{j+k}^{n-1}\right) .
\end{aligned}
$$

Due to the CFL condition (1.5), all coefficients in (3.11) are non-negative, so we obtain

$$
\begin{aligned}
\left|u_{j}^{n+1}-u_{j}^{n}\right| \leq & \lambda D_{1, j-1}^{n}\left|u_{j-1}^{n}-u_{j-1}^{n-1}\right|-\lambda D_{2, j}^{n}\left|u_{j+1}^{n}-u_{j+1}^{n-1}\right| \\
& +\left(1-\lambda\left(D_{1, j}^{n}-D_{2, j-1}^{n}\right)-2 \mu C_{\eta} a\left(\bar{v}_{j}^{n-1 / 2}\right)\left(1-w_{0}\right)\right)\left|u_{j}^{n}-u_{j}^{n-1}\right| \\
& +2 \mu C_{\eta} \sum_{\substack{k=-\eta \\
k \neq 0}}^{n} w_{-k} a\left(\bar{v}_{j+k}^{n-1 / 2}\right)\left|u_{j+k}^{n}-u_{j+k}^{n-1}\right| .
\end{aligned}
$$

Summing this over all $j \in \mathbb{Z}$ we obtain

$$
\sum_{j \in \mathbb{Z}}\left|u_{j}^{n+1}-u_{j}^{n}\right| \leq \sum_{j \in \mathbb{Z}}\left|u_{j}^{n}-u_{j}^{n-1}\right|
$$

which means that

$$
\Delta x \sum_{j \in \mathbb{Z}}\left|u_{j}^{n+1}-u_{j}^{n}\right| \leq \Delta x \sum_{j \in \mathbb{Z}}\left|u_{j}^{1}-u_{j}^{0}\right| .
$$

We need to show that the right-hand side of $(3.12)$ is $\mathcal{O}(\Delta t)$. From (3.4) for $n=0$ we obtain

$$
u_{j}^{1}-u_{j}^{0}=-\lambda \Delta_{-} F^{\mathrm{EO}}\left(u_{j}^{0}, u_{j+1}^{0}\right)+2 \mu C_{\eta} \Delta_{-} \psi_{j}^{0} \quad \text { for all } j \in \mathbb{Z} .
$$


Similarly to (3.10) we obtain

$$
\Delta_{-} F^{\mathrm{EO}}\left(u_{j}^{0}, u_{j+1}^{0}\right)=d_{2, j+1 / 2} \Delta_{+} u_{j}^{0}+d_{1, j-1 / 2} \Delta_{-} u_{j}^{0}-u_{j-1}^{0},
$$

where $d_{1, j-1 / 2}=\partial_{1} F^{\mathrm{EO}}\left(v_{j-1 / 2}, u_{j}^{0}\right) \geq 0$ and $d_{2, j-1 / 2}=\partial_{2} F^{\mathrm{EO}}\left(u_{j-1}^{0}, \tilde{v}_{j-1 / 2}\right) \leq 0$ for some $v_{j-1 / 2}, \tilde{v}_{j-1 / 2}$ between $u_{j}^{0}$ and $u_{j-1}^{0}$. Moreover, in light of (3.5) for $n=0$ we get

$$
\Delta_{-} \psi_{j}^{0}=\Delta_{-} \sum_{k=-\eta+1}^{\eta-1} Q_{k} \Delta_{+} A\left(u_{j+k}^{0}\right)
$$

so we have

$$
\Delta x \sum_{j \in \mathbb{Z}}\left|u_{j}^{1}-u_{j}^{0}\right| \leq \Delta t \sum_{j \in \mathbb{Z}}\left\{d_{1, j-1 / 2}\left|u_{j}^{0}-u_{j-1}^{0}\right|-d_{2, j+1 / 2}\left|u_{j+1}^{0}-u_{j}^{0}\right|\right\}+\Delta t \sum_{j \in \mathbb{Z}} \sum_{k=-\eta+1}^{\eta-1} \frac{Q_{k}}{\Delta x}\left|\Delta^{2} A\left(u_{j+k}^{0}\right)\right| .
$$

Now, in light of the assumption (2.4), we may write

$$
\Delta x \sum_{j \in \mathbb{Z}}\left|u_{j}^{1}-u_{j}^{0}\right| \leq 2 \Delta t\left\|f^{\prime}\right\|_{\infty} \operatorname{TV}\left(u^{0}\right)+\Delta t \sum_{k=-\eta+1}^{\eta-1} Q_{-k} \sum_{j \in \mathbb{Z}} \frac{1}{\Delta x}\left|\Delta_{-} \Delta_{+} A\left(u_{j+k}^{0}\right)\right| \leq C_{1} \Delta t
$$

with a constant $C_{1}$ that is independent of $\Delta x$ and $\Delta t$. Thus, combining (3.12) and (3.13) we obtain

$$
\sum_{j \in \mathbb{Z}}\left|u_{j}^{n+1}-u_{j}^{n}\right| \leq C_{1} \lambda
$$

which immediately implies (3.9).

Lemma 3.4. Assume that the CFL condition (1.5) is satisfied. Then there exists a constant $C_{2}$, which is independent of $\Delta t$ and $\Delta x$, such that

$$
\left|\sum_{k=-\eta+1}^{\eta-1} Q_{k}\left(A\left(u_{j+k+1}^{n}\right)-A\left(u_{j+k}^{n}\right)\right)\right| \leq C_{2} \Delta x .
$$

Proof. For each discretization $(\Delta x, \Delta t)$ and time $t^{n}$, the numerical solution values $u_{j}^{n}$ are constant outside a finite range of indices $j$. Thus, from (3.4) we deduce that

$$
\begin{aligned}
2 C_{\eta} \frac{\left|\psi_{j}^{n}\right|}{\Delta x}-\left|F^{\mathrm{EO}}\left(u_{j}^{n}, u_{j+1}^{n}\right)\right| & \leq\left|-F^{\mathrm{EO}}\left(u_{j}^{n}, u_{j+1}^{n}\right)+2 C_{\eta} \frac{\psi_{j}^{n}}{\Delta x}\right| \leq\left|\sum_{i=-\infty}^{j} \Delta_{-}\left(-F^{\mathrm{EO}}\left(u_{i}^{n}, u_{i+1}^{n}\right)+2 C_{\eta} \frac{\psi_{i}^{n}}{\Delta x}\right)\right| \\
& \leq \frac{1}{\lambda}\left|\sum_{i=-\infty}^{j}\left(u_{i}^{n+1}-u_{i}^{n}\right)\right| \leq \frac{1}{\lambda} \sum_{i \in \mathbb{Z}}\left|u_{i}^{n+1}-u_{i}^{n}\right| \leq C_{1},
\end{aligned}
$$

where $C_{1}$ is the constant of Lemma 3.3. We conclude that $\left|\psi_{j}^{n}\right| \leq C_{2} \Delta x$, where $C_{2}=\left(C_{1}+\left\|F^{\mathrm{EO}}\right\|_{\infty}\right) /\left(2 C_{\eta}\right)$, from which (3.14) follows if we take into account (3.5).

Lemma 3.5. Assume that (1.5) is satisfied, and that the mollification weights $w_{i}$ satisfy the restriction

$$
\zeta:=Q_{0}-2 \sum_{i=1}^{\eta-1} Q_{i}=w_{1}-\sum_{i=2}^{\eta}\left(i^{2}-2 i\right) w_{i}>0,
$$

where we recall that the quantities $Q_{0}, \ldots, Q_{\eta-1}$ are defined in (2.9). Then there exists a constant $C_{3}$, which is independent of $\Delta t$ and $\Delta x$, such that

$$
\left|A\left(u_{j}^{n}\right)-A\left(u_{l}^{n}\right)\right| \leq C_{3}|j-l| \Delta x \quad \text { for all } j, l \in \mathbb{Z} .
$$


Proof. We begin by setting $z_{j}^{n}:=A\left(u_{j+1}^{n}\right)-A\left(u_{j}^{n}\right)$. Since the initial datum is assumed to be constant outside a bounded interval (see (2.4)), for a given pair of discretization parameters $(\Delta x, \Delta t)$ there exists an integer $K>0$, which in general depends on $n$, such that $z_{j}^{n}=z_{-j}^{n}=0$ for $j>K$. Additionally, from the triangular inequality and Lemma 3.4 we obtain

$$
Q_{0}\left|z_{j}^{n}\right|-\sum_{i=1}^{\eta-1} Q_{i}\left|z_{j-i}^{n}\right|-\sum_{i=1}^{\eta-1} Q_{i}\left|z_{j+i}^{n}\right| \leq\left|\sum_{k=-\eta+1}^{\eta-1} Q_{-k} z_{j+k}^{n}\right| \leq C_{2} \Delta x, \quad j=-K, \ldots, K .
$$

Actually, (3.17) is valid for $j \in \mathbb{Z}$, but is trivially satisfied for $|j|>K$ since $z_{j}^{n}=0$ for these $j$. Consequently, defining the vectors

$$
\mathbf{d}^{n}:=\left(\left|z_{-K}^{n}\right|, \ldots,\left|z_{K}^{n}\right|\right)^{\mathrm{T}} \in \mathbb{R}^{2 K+1}, \quad \mathbf{e}:=(1, \ldots, 1)^{\mathrm{T}} \in \mathbb{R}^{2 K+1}
$$

and the $(2 \eta-1)$-diagonal $(2 K+1) \times(2 K+1)$-matrix

$$
\mathbf{M}=\left[\begin{array}{ccccccc}
Q_{0} & -Q_{1} & \cdots & -Q_{\eta-1} & 0 & \cdots & 0 \\
-Q_{1} & Q_{0} & \ddots & & \ddots & \ddots & \vdots \\
\vdots & \ddots & \ddots & \ddots & & \ddots & 0 \\
-Q_{\eta-1} & & \ddots & \ddots & \ddots & & -Q_{\eta-1} \\
0 & \ddots & & \ddots & \ddots & \ddots & \vdots \\
\vdots & \ddots & \ddots & & \ddots & \ddots & -Q_{1} \\
0 & \cdots & 0 & -Q_{\eta-1} & \cdots & -Q_{1} & Q_{0}
\end{array}\right]
$$

we can rewrite (3.17) as the system of inequalities $\mathbf{M d}^{n} \leq C_{2} \Delta x \mathbf{e}$, where " $\leq$ " holds in a component-wise sense. Clearly, due to its sign structure, $\mathbf{M}$ is an L-matrix. Moreover, if (3.15) is satisfied, then $\mathbf{M}$ becomes an M-matrix, which means that $\mathbf{M}^{-1}$ exists, $\mathbf{M}^{-1} \geq 0$ in a component-wise sense, and $\left\|\mathbf{M}^{-1}\right\|_{\infty} \leq \zeta^{-1}$. This implies that $\mathbf{d}^{n} \leq C_{2} \Delta x \mathbf{M}^{-1} \mathbf{e}$ (in a component-wise sense), in particular $\left\|\mathbf{d}^{n}\right\|_{\infty} \leq\left(C_{2} \zeta^{-1}\right) \Delta x$. Thus, (3.16) follows by taking $C_{3}:=C_{2} / \zeta$, and noting that $\zeta$ does not depend on $\Delta x, \Delta t$, or $K$.

Remark 3.2. We have just proved that by imposing that the mollification weights satisfy the additional condition (3.15), one can establish the spatial regularity property (3.16). For $p=3,(3.15)$ is satisfied for $\eta=1, \ldots, 5$, see Table 1 , which also shows the corresponding values of $\zeta$.

In light of Lemma 3.5 there exists a constant $C_{4}$, which is independent of $\Delta t$ and $\Delta x$, such that

$$
\lambda \sum_{j \in \mathbb{Z}} \sum_{n=0}^{N-1}\left(A\left(u_{j+1}^{n}\right)-A\left(u_{j}^{n}\right)\right)^{2} \leq C_{4} .
$$

Lemma 3.6. Under the assumptions of Lemma 3.5 there exists a constant $C_{5}$, which is independent of $\Delta t$ and $\Delta x$, such that

$$
\left|A\left(u_{j}^{n}\right)-A\left(u_{j}^{m}\right)\right| \leq C_{5}|(m-n) \Delta t|^{1 / 2} \quad \text { for all } n, m \in\{0, \ldots, N\} .
$$

The proof of Lemma 3.6 is given by the proof of [17, Lemma 4.2], which in turn is based on a technique introduced in [1]. The proof is based on an interpolation technique that exploits (3.16) and does not depend on the particular scheme being considered.

As in [1], we denote by $u_{\Delta}$ (where $\Delta=(\Delta x, \Delta t)$ ) the interpolant of degree one associated with the data points $\left\{u_{j}^{n}\right\}$. The function $u_{\Delta}$ is continuous everywhere and differentiable almost everywhere. From (3.6) in Lemma 3.2, Corollary 3.1 and Lemma 3.3 we deduce that there is a constant $C_{6}$ such that

$$
\left\|u_{\Delta}\right\|_{L^{\infty}\left(\Pi_{T}\right)}+\mathrm{TV}_{\Pi_{T}}\left(u_{\Delta}\right) \leq C_{6},
$$

while Lemmas 3.5 and 3.6 imply that there is a constant $C_{7}$ such that

$$
\left|A\left(u_{\Delta}(y, \tau)\right)-A\left(u_{\Delta}(x, t)\right)\right| \leq C_{7}\left(|x-y|+|t-\tau|^{1 / 2}+\Delta x+\Delta t^{1 / 2}\right) \quad \text { for all }(x, t),(y, \tau) \in \Pi_{T} .
$$


Lemma 3.7. Let us recall the standard notation $a \wedge b:=\min \{a, b\}$ and $a \vee b:=\max \{a, b\}$, and define

$$
\tilde{\psi}_{j}^{n}:=\sum_{k=1}^{n} \rho_{k}\left(\left|A\left(u_{j+k}^{n}\right)-A(c)\right|-\left|A\left(u_{j-k+1}^{n}\right)-A(c)\right|\right),
$$

where $\rho_{k}$ is defined as in (3.3). Then the mollified scheme (3.2) satisfies the following cell entropy inequality:

$$
\begin{aligned}
\forall c \in \mathbb{R}: \quad\left|u_{j}^{n+1}-c\right| \leq & \left|u_{j}^{n}-c\right| \\
& -\lambda \Delta_{-}\left(F^{\mathrm{EO}}\left(u_{j}^{n} \vee c, u_{j+1}^{n} \vee c\right)-F^{\mathrm{EO}}\left(u_{j}^{n} \wedge c, u_{j+1}^{n} \wedge c\right)-\frac{2 C_{\eta}}{\Delta x} \tilde{\psi}_{j}^{k}\right) .
\end{aligned}
$$

Proof. Recalling the definition of $\psi_{j}^{n}$ in (3.3), we obtain by replacing every ocurrence of $u_{j}^{n}$ in the definition of $\mathcal{S}_{j}\left(u^{n}\right)$ by $u_{j}^{n} \vee c$, where $u^{n} \vee c:=\left\{u_{j}^{n} \vee c\right\}_{j \in \mathbb{Z}}$, the identity

$$
\mathcal{S}_{j}\left(u^{n} \vee c\right)=u_{j}^{n} \vee c-\lambda \Delta_{-}\left(F^{\mathrm{EO}}\left(u_{j}^{n} \vee c, u_{j+1}^{n} \vee c\right)-\frac{2 C_{\eta}}{\Delta x} \sum_{k=1}^{n} \rho_{k}\left(A\left(u_{j+k}^{n} \vee c\right)-A\left(u_{j-k+1}^{n} \vee c\right)\right)\right) .
$$

The same identity holds if every " $\vee$ " is replaced by " $\wedge$ " and we define $u^{n} \wedge c:=\left\{u_{j}^{n} \wedge c\right\}_{j \in \mathbb{Z} \text {. Subtracting }}$ the second version from the first, we obtain

$$
\begin{aligned}
& \mathcal{S}_{j}\left(u^{n} \vee c\right)-\mathcal{S}_{j}\left(u^{n} \wedge c\right) \\
& =\left|u_{j}^{n}-c\right|-\lambda \Delta_{-}\left(F^{\mathrm{EO}}\left(u_{j}^{n} \vee c, u_{j+1}^{n} \vee c\right)-F^{\mathrm{EO}}\left(u_{j}^{n} \wedge c, u_{j+1}^{n} \wedge c\right)\right) \\
& \quad-\lambda \Delta_{-}\left(-\frac{2 C_{\eta}}{\Delta x} \sum_{k=1}^{n} \rho_{k}\left(A\left(u_{j+k}^{n} \vee c\right)-A\left(u_{j+k}^{n} \wedge c\right)-\left(A\left(u_{j-k+1}^{n} \vee c\right)-A\left(u_{j-k+1}^{n} \wedge c\right)\right)\right)\right) .
\end{aligned}
$$

Since $A$ is non-decreasing, we can rewrite this as

$$
\begin{aligned}
\mathcal{S}_{j}\left(u^{n} \vee c\right)-\mathcal{S}_{j}\left(u^{n} \wedge c\right)= & \left|u_{j}^{n}-c\right|-\lambda \Delta_{-}\left(F^{\mathrm{EO}}\left(u_{j}^{n} \vee c, u_{j+1}^{n} \vee c\right)-F^{\mathrm{EO}}\left(u_{j}^{n} \wedge c, u_{j+1}^{n} \wedge c\right)\right) \\
& +\lambda \frac{2 C_{\eta}}{\Delta x} \Delta_{-} \tilde{\psi}_{j}^{n} .
\end{aligned}
$$

On the other hand, the monotonicity of the scheme implies that

$$
\mathcal{S}_{j}\left(u^{n} \vee c\right)-\mathcal{S}_{j}\left(u^{n} \wedge c\right) \geq \mathcal{S}_{j}\left(u^{n}\right) \vee c-\mathcal{S}_{j}\left(u^{n}\right) \wedge c=\left|u_{j}^{n+1}-c\right| .
$$

Combining (3.22) and (3.23) we obtain the desired entropy inequality (3.21).

Theorem 3.2. Assume that $\Delta t$ and $\Delta x$ satisfy the CFL condition (1.5), that the weights $w_{-\eta}, \ldots, w_{\eta}$ of the discrete mollification operator satisfy the restriction (3.15), and that the initial datum $u_{0}$ satisfies (2.4). Then the interpolated approximate solution $u_{\Delta}$ obtained from the mollified scheme (3.2) converges in the strong topology of $L^{1}\left(\Pi_{T}\right)$ to an entropy solution of (1.1), (1.2).

Proof. Due to the embedding of $L^{\infty}\left(\Pi_{T}\right) \cap B V\left(\Pi_{T}\right)$ in $L^{1}\left(Q_{T}\right)$ ([22]; see [1]), we deduce from (3.19) that there exists a sequence $\left\{\Delta_{i}\right\}_{i \in \mathbb{N}}$ with $\Delta_{i} \rightarrow 0$ for $i \rightarrow \infty$ and a function $u \in L^{\infty}\left(\Pi_{T}\right) \cap B V\left(\Pi_{T}\right)$ such that $u_{\Delta} \rightarrow u$ a.e. on $\Pi_{T}$. Moreover, in light of (3.20) the Arzelà-Ascoli theorem implies that $A\left(u_{\Delta}\right) \rightarrow A(u)$ uniformly on $\Pi_{T}$, and we have that $A(u) \in C^{1,1 / 2}\left(\Pi_{T}\right)$.

It remains to prove that $u$ satisfies the entropy inequality (2.1). This can be done by a standard LaxWendroff-type argument; namely, we choose a non-negative test function $\phi \in C^{\infty}\left(\Pi_{T}\right)$ with compact support on $\mathbb{R} \times[0, T)$, multiply the discrete entropy inequality (3.21) by $\Delta x \phi_{j}^{n}$, where $\phi_{j}^{n}=\phi\left(x_{j}, t^{n}\right)$, sum the result over all $j$ and $n$, apply "summation by parts", and let $\Delta \downarrow 0$. Details (cf. e.g. [1, 15]) will be omitted here, but we mention that the "summation by parts" for the discretization of the diffusive terms in (3.21) can be done as follows:

$$
\Delta x \sum_{j \in \mathbb{Z}} \sum_{n=0}^{N-1} \lambda \phi_{j}^{n} \Delta_{-}\left(\frac{2 C_{\eta}}{\Delta x} \tilde{\psi}_{j}^{n}\right)=\Delta x \Delta t \sum_{n=0}^{N-1} \frac{2 C_{\eta}}{\Delta x^{2}} \sum_{j \in \mathbb{Z}} \phi_{j}^{n}\left(\sum_{i=-\eta}^{\eta} w_{-i}\left|A\left(u_{j+i}^{n}\right)-A(c)\right|-\left|A\left(u_{j}^{n}\right)-A(c)\right|\right)
$$




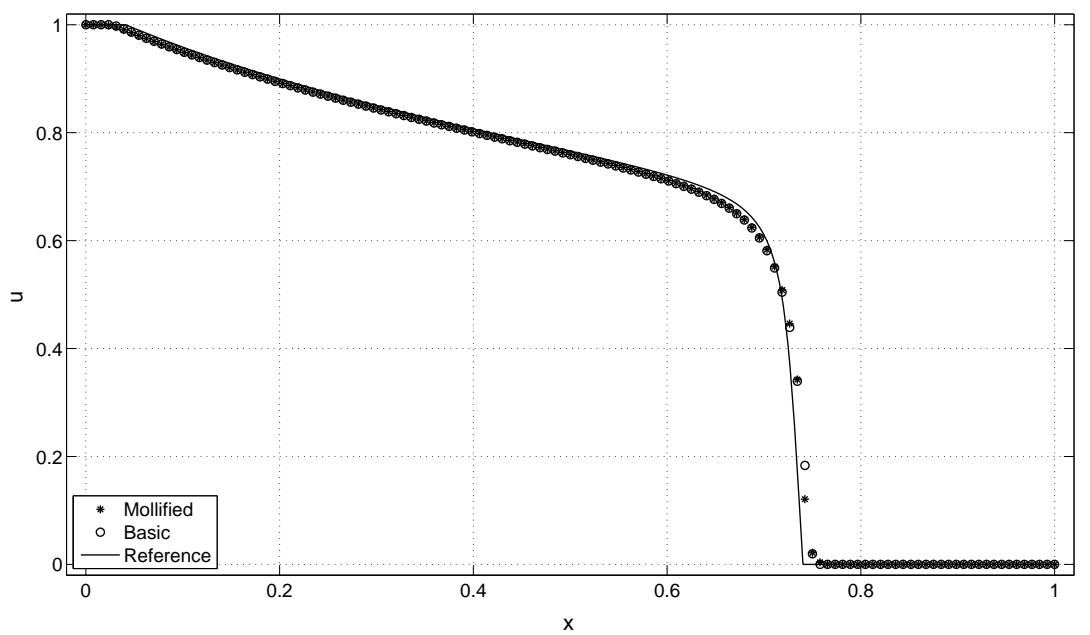

Figure 2. Example 1: numerical solution at $T=0.5$ calculated by the basic scheme $(2.3)$ and the mollified scheme (3.2) with $\eta=5$ with $\Delta x=1 / 128$. The solid line is the reference solution corresponding to $\Delta x=1 / 4096$.

$$
\begin{aligned}
& =\Delta x \Delta t \sum_{n=0}^{N-1} \sum_{j \in \mathbb{Z}}\left|A\left(u_{j}^{n}\right)-A(c)\right| \frac{2 C_{\eta}}{\Delta x^{2}}\left(\sum_{i=-\eta}^{\eta} w_{-i} \phi_{j-i}^{n}-\phi_{j}^{n}\right) \\
& =\Delta x \Delta t \sum_{n=0}^{N-1} \sum_{j \in \mathbb{Z}}\left|A\left(u_{j}^{n}\right)-A(c)\right| \phi_{x x}\left(x_{j}, t^{n}\right)+\mathcal{O}\left(\Delta x^{2}\right),
\end{aligned}
$$

where the last equality follows from applying (3.1) to the smooth test function $\phi$.

\section{Numerical Examples}

In this section we present numerical solutions of some test problems to evaluate the performance of the mollified scheme (3.2). For comparison purposes we will use the basic scheme (2.3) as reference. In both cases, the time step $\Delta t$ is selected by considering equality in the respective CFL conditions (1.4) and (1.5) with the right-hand sides set to 0.98 . Thus, for the mollified scheme we employ

$$
\Delta t=0.98 \frac{\Delta x^{2}}{\Delta x\left\|f^{\prime}\right\|_{\infty}+2 \varepsilon_{\eta}\|a\|_{\infty}} .
$$

In all examples a reference solution was computed using the basic scheme on a very fine grid. This reference solution was then used for approximating the error of the schemes on coarser grids. More precisely, the relative $L^{1}$-error was approximated by the quantity

$$
\left(\sum_{i=1}^{M}\left|U^{n}\left(x_{j}\right)\right|\right)^{-1} \sum_{i=1}^{M}\left|u_{j}^{n}-U^{n}\left(x_{j}\right)\right|
$$

where $M$ is the total number of grid points, $u_{j}^{n}$ is the computed solution at $x_{j}$ in the simulated time $t^{n}$ and $U^{n}\left(x_{j}\right)$ is value of the reference solution at the same time $t^{n}$ in the grid point $x_{j}$. The grids were built in such a way that no spatial interpolation is needed to evaluate $U^{n}\left(x_{j}\right)$. However, the last time step was fixed for both methods so that the desired final time is attained exactly. 
MOLLIFIED SCHEMES FOR STRONGLY DEGENERATE PARABOLIC EQUATIONS

\begin{tabular}{|c|c|c|c|c|c|c|}
\hline \multirow[b]{2}{*}{$1 / \Delta x$} & \multicolumn{3}{|c|}{ Basic scheme (2.3) } & \multicolumn{3}{|c|}{ Mollified Scheme (3.2) with $\eta=3$} \\
\hline & $L^{1}$-error & conv. rate & CPU time $[\mathrm{s}]$ & $L^{1}$-error & conv. rate & CPU time $[\mathrm{s}$ \\
\hline 64 & $2.6762 \mathrm{e}-2$ & - & 0.0083 & $2.6105 \mathrm{e}-2$ & - & 0.0107 \\
\hline 128 & $1.5390 \mathrm{e}-2$ & 0.7982 & 0.0286 & $1.4932 \mathrm{e}-2$ & 0.8059 & 0.0384 \\
\hline 256 & $8.5957 \mathrm{e}-3$ & 0.8403 & 0.1074 & $8.3709 \mathrm{e}-3$ & 0.8349 & 0.1330 \\
\hline 512 & $4.5905 \mathrm{e}-3$ & 0.9050 & 0.5497 & $4.5075 \mathrm{e}-3$ & 0.8931 & 0.6497 \\
\hline 1024 & $2.0265 \mathrm{e}-3$ & 1.1797 & 3.4742 & $1.9997 \mathrm{e}-3$ & 1.1725 & 3.8324 \\
\hline & \multicolumn{3}{|c|}{ Mollified Scheme (3.2) with $\eta=5$} & \multicolumn{3}{|c|}{ Mollified Scheme (3.2) with $\eta=8$} \\
\hline $1 / \Delta x$ & $L^{1}$-error & conv. rate & CPU time $[\mathrm{s}]$ & $L^{1}$-error & conv. rate & CPU time $[\mathrm{s}$ \\
\hline 64 & $2.5327 \mathrm{e}-2$ & - & 0.0101 & $2.5055 \mathrm{e}-2$ & - & 0.0089 \\
\hline 128 & $1.4287 \mathrm{e}-2$ & 0.8259 & 0.0308 & $1.4133 \mathrm{e}-2$ & 0.8260 & 0.0319 \\
\hline 256 & $7.9698 \mathrm{e}-3$ & 0.8420 & 0.1007 & $7.6883 \mathrm{e}-3$ & 0.8783 & 0.0893 \\
\hline 512 & $4.3271 \mathrm{e}-3$ & 0.8811 & 0.4380 & $4.1141 \mathrm{e}-3$ & 0.9020 & 0.3075 \\
\hline 1024 & $1.9335 \mathrm{e}-3$ & 1.1622 & 2.3848 & $1.8279 \mathrm{e}-3$ & 1.1704 & 1.4976 \\
\hline
\end{tabular}

TABLE 3. Example 1: approximate relative $L^{1}$ errors and CPU times for the basic scheme (2.3) and the mollified scheme (3.2) with $\eta=3, \eta=5$ and $\eta=8$, for the simulated time $T=0.5$.
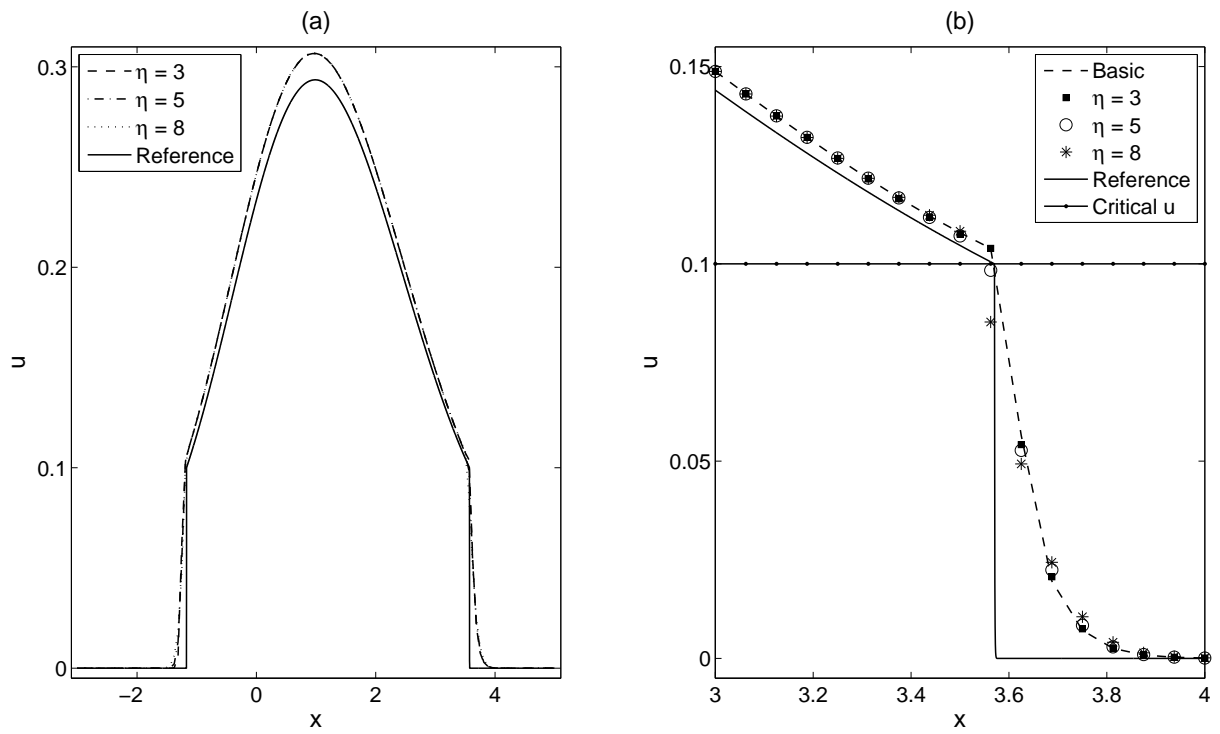

Figure 3. Example 2: (a) numerical solution at $T=1$ calculated by the mollified scheme (3.2) with $\eta=3, \eta=5$ and $\eta=8$ for $\Delta x=1 / 16$, (b) enlarged view of a portion of (a), showing also the solution obtained by the basic scheme (2.3) with $\Delta x=1 / 16$. In both plots, the solid line is the reference solution calculated by the basic scheme (2.3) with $\Delta x=1 / 2048$.

Example 1 (Buckley-Leverett Problem). Consider the saturation equation given by (1.1) with

$$
f(u)=\frac{u^{2}}{u^{2}+(1-u)^{2}}, \quad a(u)=4 \varepsilon u(1-u),
$$



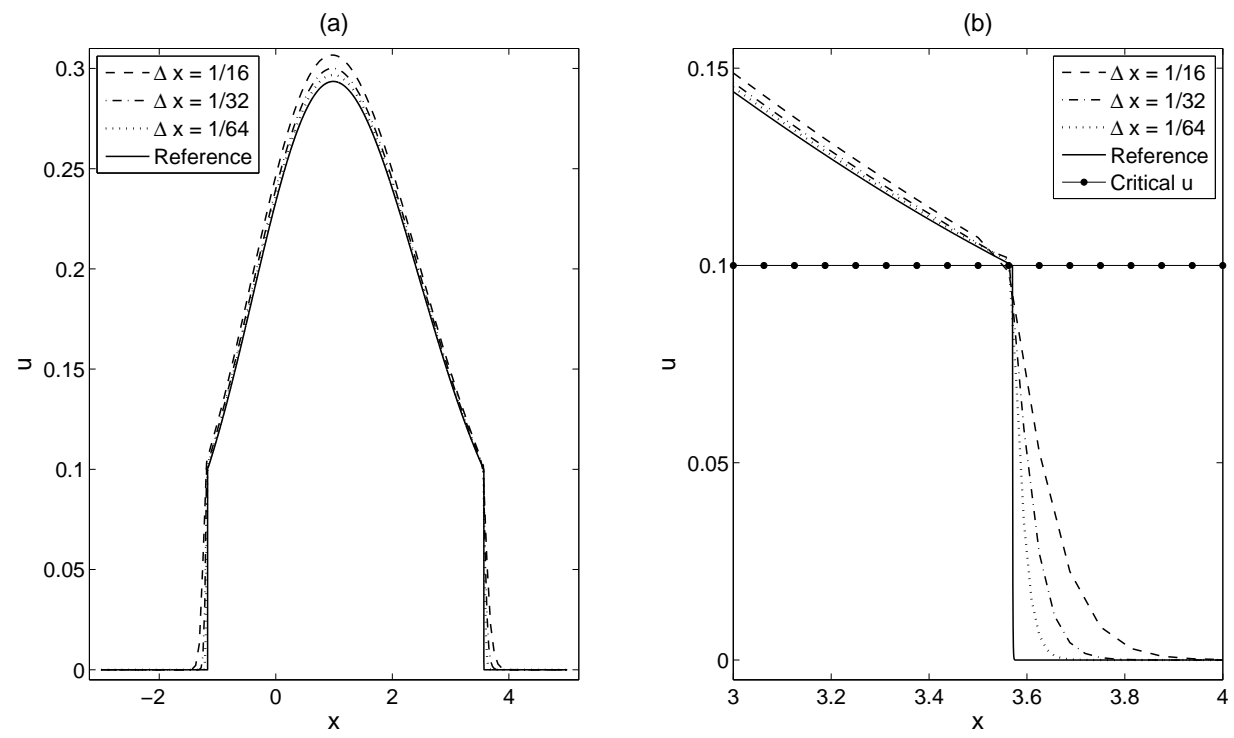

Figure 4. Example 2: (a) numerical solution at $T=1$ calculated by the mollified scheme (3.2) with $\eta=5$ and several values of $\Delta x$, (b) enlarged view of a portion of (a). In both plots, the solid line is the reference solution calculated by the basic scheme (2.3) with $\Delta x=1 / 2048$.

\begin{tabular}{|c|c|c|c|c|c|c|}
\hline \multirow[b]{2}{*}{$1 / \Delta x$} & \multicolumn{3}{|c|}{ Basic scheme (2.3) } & \multicolumn{3}{|c|}{ Mollified Scheme (3.2) with $\eta=3$} \\
\hline & $L^{1}$-error & conv. rate & CPU time $[\mathrm{s}]$ & $L^{1}$-error & conv. rate & CPU time $[\mathrm{s}]$ \\
\hline 16 & $6.1071 \mathrm{e}-2$ & - & 0.0396 & $6.1071 \mathrm{e}-2$ & - & 0.0427 \\
\hline 32 & $2.9857 \mathrm{e}-2$ & 1.0324 & 0.1950 & $2.9857 \mathrm{e}-2$ & 1.0324 & 0.2197 \\
\hline 64 & $1.5843 \mathrm{e}-2$ & 0.9142 & 1.1440 & $1.5843 \mathrm{e}-2$ & 0.9142 & 1.2686 \\
\hline 128 & $7.2442 \mathrm{e}-3$ & 1.1289 & 7.8796 & $7.2514 \mathrm{e}-3$ & 1.1275 & 8.3968 \\
\hline 256 & $3.3381 \mathrm{e}-3$ & 1.1178 & 57.624 & $3.3466 \mathrm{e}-3$ & 1.1156 & 59.729 \\
\hline 512 & $1.3787 \mathrm{e}-3$ & 1.2757 & 411.29 & $1.3855 \mathrm{e}-3$ & 1.2723 & 426.62 \\
\hline & \multicolumn{3}{|c|}{ Mollified Scheme (3.2) with $\eta=5$} & \multicolumn{3}{|c|}{ Mollified Scheme (3.2) with $\eta=8$} \\
\hline $1 / \Delta x$ & $L^{1}$-error & conv. rate & CPU time $[\mathrm{s}]$ & $L^{1}$-error & conv. rate & CPU time $[\mathrm{s}]$ \\
\hline 16 & $6.1339 \mathrm{e}-2$ & - & 0.0299 & $6.2973 \mathrm{e}-2$ & - & 0.0149 \\
\hline 32 & $2.9857 \mathrm{e}-2$ & 1.0387 & 0.1282 & $3.0526 \mathrm{e}-2$ & 1.0447 & 0.0749 \\
\hline 64 & $1.5843 \mathrm{e}-2$ & 0.9142 & 0.7390 & $1.5954 \mathrm{e}-2$ & 0.9361 & 0.3798 \\
\hline 128 & $7.3764 \mathrm{e}-3$ & 1.1029 & 4.8372 & $7.5281 \mathrm{e}-3$ & 1.0836 & 2.4967 \\
\hline 256 & $3.4070 \mathrm{e}-3$ & 1.1144 & 34.608 & $3.4808 \mathrm{e}-3$ & 1.1129 & 17.937 \\
\hline 512 & $1.4149 \mathrm{e}-3$ & 1.2678 & 251.65 & $1.4512 \mathrm{e}-3$ & 1.2622 & 131.28 \\
\hline
\end{tabular}

TABLe 4. Example 2: approximate relative $L^{1}$ errors and CPU times for the reference scheme (2.3) and the mollified scheme (3.2) with $\eta=3, \eta=5$ and $\eta=8$, for the simulated time $T=1$.

on the domain $[0,1]$ with initial data $u_{0}(x)=1$ for $x<0.1$ and $u_{0}(x)=0$ otherwise, and $\varepsilon=0.01$. This example was solved by an operator splitting method in [23].

Here, the boundary conditions used for the computation of the numerical solution were $u(0, t)=1$ and $u(1, t)=0$. For computing the discrete mollification of $A(u)$ a reflection of the data in the interior was 

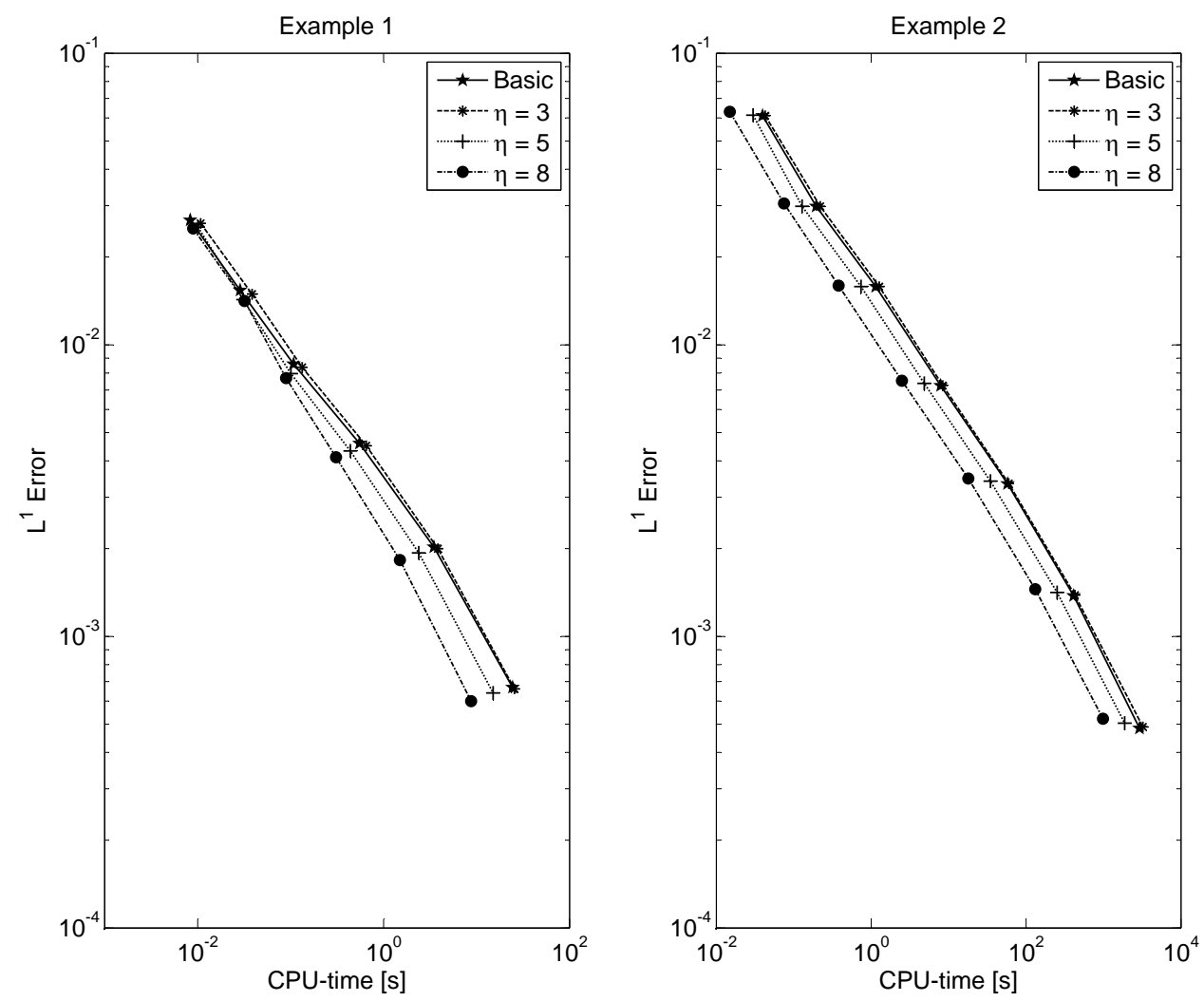

Figure 5. Examples 1 (left) and 2 (right): approximate $L^{1}$ error of the basic scheme $(2.3)$ and the of mollified scheme (3.2) with several values of $\eta$ versus the CPU time (in seconds), for a simulated time $T=0.5$ (left) and $T=1$ (right) with $\Delta x=2^{-n}$ for $n=6, \ldots, 11$ (left) and $\Delta x=2^{-n}$ for $n=4, \ldots, 10$ (right).

implemented. For details on the treatment of boundary conditions with discrete mollification we refer to [3]. The results obtained by the basic scheme (2.3) and the mollified version (3.2) are summarized in Table 3 and illustrated in Figures 2-5. The error is computed using as reference solution the result of (2.3) on a very fine grid with $\Delta x=1 / 4096$. For the mollified scheme we used $\eta=3, \eta=5$ and $\eta=8$.

Example 2. Next, we consider (1.1) with

$$
f(u)=u(1-u), \quad a(u)=\left\{\begin{array}{ll}
0 & \text { for } u \leq 0.1, \\
1 & \text { for } u>0.1,
\end{array} \quad u_{0}(x)= \begin{cases}1 & \text { for } x \in[0,1], \\
0 & \text { otherwise }\end{cases}\right.
$$

Under these assumptions, (1.1) turns into an algebraically simplified version of a diffusively corrected kinematic-wave traffic model [24]. We solve the problem up to $T=1$. Again we choose $\eta=3, \eta=5$ and $\eta=8$. In this case, the boundary conditions for $u$ and the discrete mollification of $A(u)$ were of type Dirichlet, $u(-3, t)=u(5, t)=0$. However, $x=-3$ and $x=5$ are far away from the actual support of the numerical solution, so the numerical solution coincides with that of the initial value problem (1.1), (1.2). See Table 4 for approximate errors, CPU times and convergence rates, and Figures 3 and 4 for numerical solutions where we illustrate the effects of different values of $\eta$ at a given fixed spatial discretization and of reducing $\Delta x$ for a fixed value of $\eta$, respectively. Figure 5 displays the approximate $L^{1}$ error versus the $C P U$ time for the reference scheme and the mollified version with several values of $\eta$. 
(a) $\mathrm{T}=800 \mathrm{~s}$

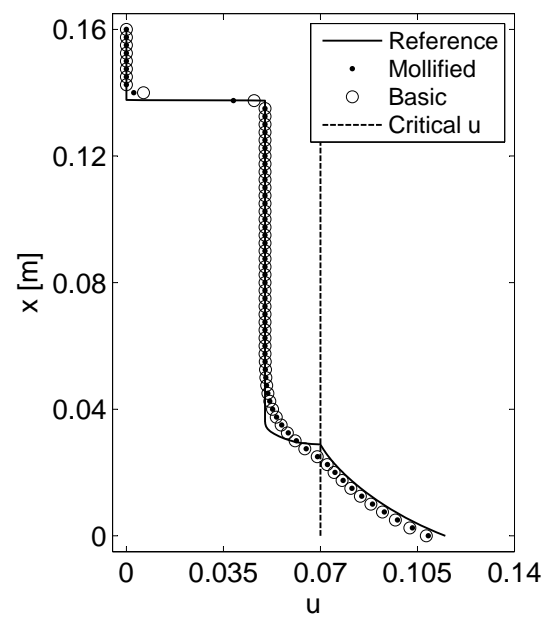

(c) $\mathrm{T}=2400 \mathrm{~s}$

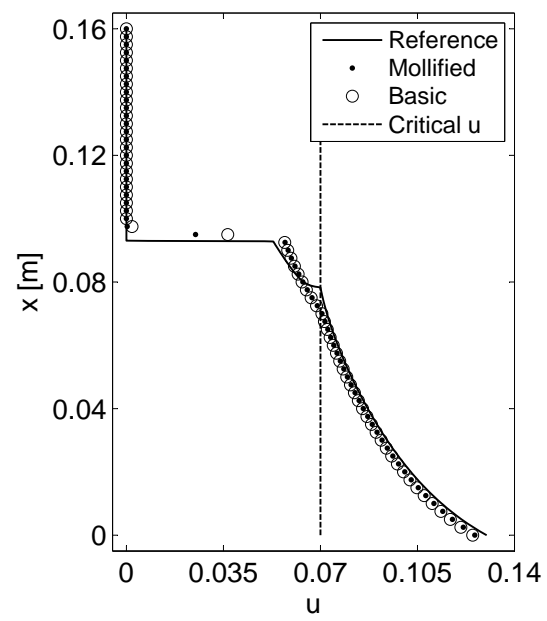

(b) $\mathrm{T}=1600 \mathrm{~s}$

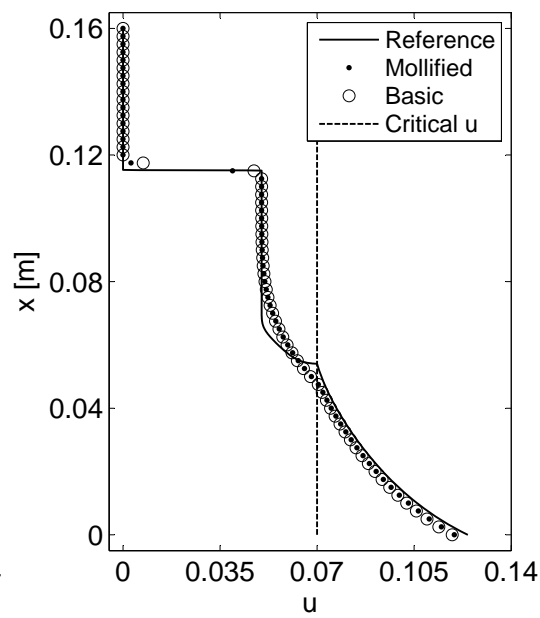

(d) $\mathrm{T}=4000 \mathrm{~s}$

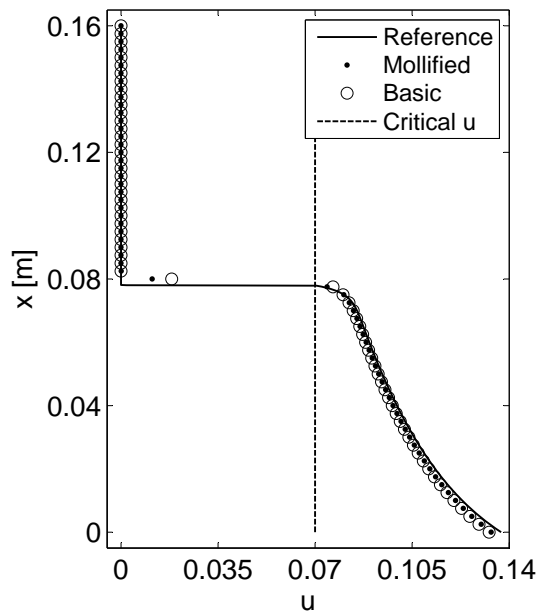

Figure 6. Example 3: numerical solution four different simulated times calculated by the basic scheme (2.3) and the mollified scheme (3.2) with $\eta=5$ and $\Delta x=L / 64$. In all plots, the solid line is the reference solution calculated by the basic scheme (2.3) with $\Delta x=L / 2048$.

Example 3 (Sedimentation). One of the main applications of strongly degenerate parabolic equations is a model of sedimentation-consolidation processes of solid-liquid suspensions, see e.g. [25], where the functions $f(u)$ and $A(u)$ model the effects of hindered settling and sediment compressibility, respectively, of a suspension of local solids volume fraction $u$. Our example is the same as [26, Sect. 4.2.1] and [27, Example 1] (where this problem was treated by an adaptive multiresolution technique), so results may be compared. We 

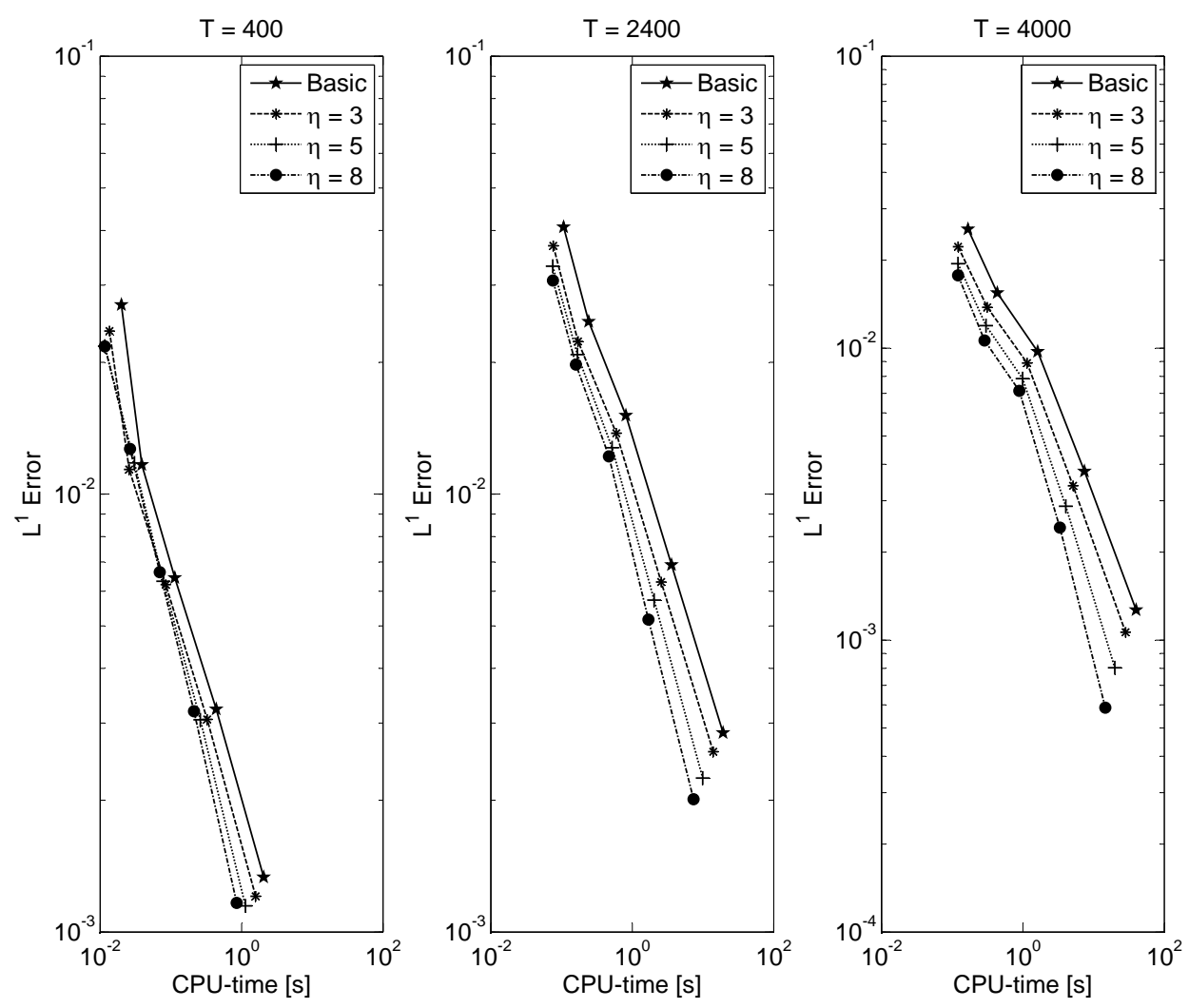

FiguRE 7. Example 3: approximate $L^{1}$ error of the basic scheme (2.3) and the of mollified scheme (3.2) with several values of $\eta$ versus the CPU time (in seconds), for the simulated time $T=400 \mathrm{~s}$ (left), $T=2400 \mathrm{~s}$ (middle) and $T=4000 \mathrm{~s}$ (right) with $\Delta x=2^{-n} L$ for $n=6, \ldots, 10$.

consider (1.1) with the choice

$$
f(u)=\left\{\begin{array}{ll}
v_{\infty} u(1-u)^{C} & \text { for } 0<u<u_{\max }, \\
0 & \text { otherwise },
\end{array} \quad \text { with parameters } v_{\infty}<0 \text { and } C>1,\right.
$$

and a diffusion function $A(u)$ defined by (1.3) with

$$
a(u)=\frac{f(u) \sigma_{\mathrm{e}}^{\prime}(u)}{\Delta_{\varrho} g u}, \quad \text { where } \sigma_{\mathrm{e}}(u)=\left\{\begin{array}{ll}
0 & \text { for } 0<u \leq u_{\mathrm{c}}, \\
\sigma_{0}\left[\left(u / u_{\mathrm{c}}\right)^{\beta}-1\right] & \text { for } u>u_{\mathrm{c}},
\end{array} \sigma_{0}>0, \quad \beta>1,\right.
$$

where $\Delta_{\varrho}$ and $g$ are constants and $u_{\mathrm{c}}$ (the so-called critical concentration), $\beta$ and $\sigma_{0}$ are parameters. If $\beta$ is an integer, then $A(u)$ can be evaluated in closed form as follows:

$$
A(u)=\left\{\begin{array}{ll}
0 & \text { for } 0<u \leq u_{\mathrm{c}}, \\
\mathcal{A}(u)-\mathcal{A}\left(u_{\mathrm{c}}\right) & \text { for } u>u_{\mathrm{c}},
\end{array} \quad \text { where } \quad \mathcal{A}(u)=\frac{v_{\infty} \sigma_{0}}{\Delta_{\varrho} g u_{\mathrm{c}}^{\beta}} \sum_{k=1}^{\beta}\left(\prod_{l=1}^{k} \frac{\beta+1-l}{C+l}\right)(1-u)^{C+k} u^{\beta-k} .\right.
$$

We consider $x \in[0, L]$ and time $t \in[0, T]$. The initial and boundary conditions are of the form

$$
u(x, 0)=u_{0}(x) \quad \text { for } x \in[0, L], \quad f(u)-A(u)_{x}=0, \quad \text { for } x=0 \text { and } x=L .
$$


(a) $T=400 \mathrm{~s}$

\begin{tabular}{|c|c|c|c|c|c|c|}
\hline \multirow[b]{2}{*}{$1 / \Delta x$} & \multicolumn{3}{|c|}{ Basic scheme (2.3) } & \multicolumn{3}{|c|}{ Mollified Scheme (3.2) with $\eta=3$} \\
\hline & $L^{1}$-error & conv. rate & CPU time $[\mathrm{s}]$ & $L^{1}$-error & conv. rate & CPU time $[\mathrm{s}]$ \\
\hline 64 & $2.7063 \mathrm{e}-2$ & - & 0.0199 & $2.3574 \mathrm{e}-2$ & - & 0.0135 \\
\hline 128 & $1.1672 \mathrm{e}-2$ & 1.2133 & 0.0388 & $1.1381 \mathrm{e}-2$ & 1.0506 & 0.0257 \\
\hline 256 & $6.4428 \mathrm{e}-3$ & 0.8572 & 0.1134 & $6.2139 \mathrm{e}-3$ & 0.8730 & 0.0845 \\
\hline 512 & $3.2280 \mathrm{e}-3$ & 0.9970 & 0.4365 & $3.0598 \mathrm{e}-3$ & 1.0221 & 0.3255 \\
\hline $1 / \Delta x$ & \multicolumn{3}{|c|}{ Mollified Scheme (3.2) with $\eta=5$} & \multicolumn{3}{|c|}{ Mollified Scheme (3.2) with $\eta=8$} \\
\hline 64 & $2.1772 \mathrm{e}-2$ & - & 0.0116 & $2.1746 \mathrm{e}-2$ & & 0.0116 \\
\hline 128 & $1.1781 \mathrm{e}-2$ & 0.8860 & 0.0305 & $1.2684 \mathrm{e}-2$ & 0.7777 & 0.0264 \\
\hline 256 & $6.3253 \mathrm{e}-3$ & 0.8972 & 0.0779 & $6.6323 \mathrm{e}-3$ & 0.9354 & 0.0695 \\
\hline 512 & $3.0530 \mathrm{e}-3$ & 1.0509 & 0.2604 & $3.1926 \mathrm{e}-3$ & 1.0548 & 0.2117 \\
\hline
\end{tabular}

(b) $T=2400 \mathrm{~s}$

\begin{tabular}{|c|c|c|c|c|c|c|}
\hline \multirow[b]{2}{*}{$1 / \Delta x$} & \multicolumn{3}{|c|}{ Basic scheme (2.3) } & \multicolumn{3}{|c|}{ Mollified Scheme (3.2) with $\eta=3$} \\
\hline & $L^{1}$-error & conv. rate & CPU time $[\mathrm{s}]$ & $L^{1}$-error & conv. rate & CPU time $[\mathrm{s}]$ \\
\hline 64 & $4.0720 \mathrm{e}-2$ & - & 0.1070 & $3.6909 \mathrm{e}-2$ & & 0.0763 \\
\hline 128 & $2.4783 \mathrm{e}-2$ & 0.7164 & 0.2419 & $2.2321 \mathrm{e}-2$ & 0.7256 & 0.1711 \\
\hline 256 & $1.5127 \mathrm{e}-2$ & 0.7122 & 0.8116 & $1.3774 \mathrm{e}-2$ & 0.6965 & 0.5954 \\
\hline 512 & $6.8969 \mathrm{e}-3$ & 1.1331 & 3.5935 & $6.3001 \mathrm{e}-3$ & 1.1285 & 2.5624 \\
\hline $1 / \Delta x$ & \multicolumn{3}{|c|}{ Mollified Scheme (3.2) with $\eta=5$} & \multicolumn{3}{|c|}{ Mollified Scheme (3.2) with $\eta=8$} \\
\hline 64 & $3.3140 \mathrm{e}-2$ & - & 0.0751 & $3.0733 \mathrm{e}-2$ & - & 0.0757 \\
\hline 128 & $2.0822 \mathrm{e}-2$ & 0.6705 & 0.1676 & $1.9756 \mathrm{e}-2$ & 0.6375 & 0.1598 \\
\hline 256 & $1.2763 \mathrm{e}-2$ & 0.7061 & 0.5181 & $1.2194 \mathrm{e}-2$ & 0.6961 & 0.4673 \\
\hline 512 & $5.7278 \mathrm{e}-3$ & 1.1559 & 2.0387 & $5.1709 \mathrm{e}-3$ & 1.2377 & 1.6909 \\
\hline
\end{tabular}

(c) $T=4000 \mathrm{~s}$

\begin{tabular}{|c|c|c|c|c|c|c|}
\hline \multirow[b]{2}{*}{$1 / \Delta x$} & \multicolumn{3}{|c|}{ Basic scheme (2.3) } & \multicolumn{3}{|c|}{ Mollified Scheme (3.2) with $\eta=3$} \\
\hline & $L^{1}$-error & conv. rate & CPU time $[\mathrm{s}]$ & $L^{1}$-error & conv. rate & CPU time $[\mathrm{s}]$ \\
\hline 64 & $2.5560 \mathrm{e}-2$ & - & 0.1670 & $2.2249 \mathrm{e}-2$ & - & 0.1209 \\
\hline 128 & $1.5463 \mathrm{e}-2$ & 0.7251 & 0.4342 & $1.3797 \mathrm{e}-2$ & 0.6894 & 0.3133 \\
\hline 256 & $9.7331 \mathrm{e}-3$ & 0.6679 & 1.6177 & $8.8938 \mathrm{e}-3$ & 0.6335 & 1.1459 \\
\hline 512 & $3.7879 \mathrm{e}-3$ & 1.3615 & 7.4223 & $3.3775 \mathrm{e}-3$ & 1.3968 & 5.1181 \\
\hline $1 / \Delta x$ & \multicolumn{3}{|c|}{ Mollified Scheme (3.2) with $\eta=5$} & \multicolumn{3}{|c|}{ Mollified Scheme (3.2) with $\eta=8$} \\
\hline 64 & $1.9460 \mathrm{e}-2$ & - & 0.1208 & $1.7743 \mathrm{e}-2$ & - & 0.1211 \\
\hline 128 & $1.1946 \mathrm{e}-2$ & 0.7039 & 0.3008 & $1.0622 \mathrm{e}-2$ & 0.7402 & 0.2863 \\
\hline 256 & $7.8624 \mathrm{e}-3$ & 0.6034 & 0.9887 & $7.1371 \mathrm{e}-3$ & 0.5737 & 0.8896 \\
\hline 512 & $2.8738 \mathrm{e}-3$ & 1.4520 & 4.0391 & $2.4282 \mathrm{e}-3$ & 1.5555 & 3.3389 \\
\hline
\end{tabular}

TABLE 5. Example 3: approximate relative $L^{1}$ errors and CPU times for the reference scheme (2.3) and the mollified scheme (3.2) with $\eta=3, \eta=5$ and $\eta=8$, for the simulated times (a) $T=400 \mathrm{~s}$, (b) $T=2400 \mathrm{~s}$ and (c) $T=4000 \mathrm{~s}$.

The values for the parameters are: $v_{\infty}=-2.7 \times 10^{-4} \mathrm{~m} / \mathrm{s}, u_{\mathrm{c}}=0.07, u_{\max }=0.5, C=21.5, \sigma_{0}=1.2 \mathrm{~Pa}$, $\beta=5, \Delta_{\varrho}=1660 \mathrm{~kg} / \mathrm{m}^{3}, L=0.16 \mathrm{~m}, g=9.81 \mathrm{~m} / \mathrm{s}^{2}$ and the initial condition $u_{0}(x)=0.05$ for $x \in[0, L]$. The implementation of the boundary conditions for $u$ is the same in [26, Sect. 4.2.1] and [27, Example 1]. 
For the discrete mollification of $A\left(u^{n}\right)$, we obtained a linear extrapolation at the borders. For instance at $x=0$, we use the knowledge of $A\left(u_{0}^{n}\right)$ and $A\left(u_{0}^{n}\right)_{x}=f\left(u_{0}^{n}\right)$ for getting an interpolating straight line with slope $f\left(u_{0}^{n}\right)$. See Table 5 and Figures 6 and 7 for results.

\section{Conclusions}

The convergence analysis shows that standard compactness and entropicity arguments for finite difference schemes for non-linear first-order conservation laws and strongly degenerate parabolic equations can be applied to establish convergence of (3.2) to the entropy solution of (1.1), (1.2) under a CFL condition that allows a larger time step than the basic (unmollified) scheme. Although the convergence statement holds only for $\eta \leq 5$ (when $p=3$ ), encouraging numerical results were also obtained in the case $\eta>5$, as illustrated above with $\eta=8$. A possibly sharper bound in Lemma 3.5 could lead to a less restrictive convergence condition than (3.15). We have kept here the arguments fairly simple, and limited ourselves to one set of mollification weights, namely those obtained in Table 1 for $p=3$; this value of $p$ is also employed in $[3,10]$. Variations of $p$ and of the corresponding weights could equally turn out more favorable conditions of satisfaction of (3.15).

Overall, the results of the numerical solutions look encouraging and in the cases of Examples 1 and 3 illustrate that the mollified scheme works well also for problems with boundary conditions, which we have not included in our convergence analysis (see [15]). The computations have been performed with the maximal time step allowed by the corresponding CFL conditions for the basic scheme and its mollified versions. One should keep in mind that the mollified schemes permit a larger time step, but the evaluation of the discretization of $A(u)_{x x}$ for the mollified scheme is algebraically slightly more involved that that for the basic scheme. In fact, in Examples 1 and 2 a significant gain in CPU time is achieved only for $\eta=5$ and $\eta=8$. In all examples, the approximate errors for the basic and mollified schemes at a given discretization are similar. Probably the resulting speed-up is not as good as for implicit versions of the basic scheme, but the mollification-based option presented herein is easy to implement and avoids, for example, the necessity to solve systems of nonlinear equations that appear with implicit schemes. Furthermore, we mention that in any case a numerical solution calculated by the mollified scheme on a portion of $\Pi_{T}$ requires less storage space than the solution obtained from the basic scheme. This makes it potentially interesting to use the mollified scheme (3.2) as the basic forward solver for parameter identification problems (see [20]), in which the coefficients of the so-called adjoint (backward-in-time) scheme depend on the properly stored solution of the direct (forward-in-time) problem.

\section{ACKNOWLEDGEMENTS}

CDA and RB acknowledge support by Fondap in Applied Mathematics, project 15000001, BASAL project CMM, Universidad de Chile and Centro de Investigación en Ingeniería Matemática ( $\mathrm{CI}^{2} \mathrm{MA}$ ), Universidad de Concepción, and project AMIRA P996/INNOVA 08CM01-17 "Instrumentación y Control de Espesadores". In addition, RB is supported by Fondecyt project 1090456 and CDA by Universidad Nacional de Colombia, DIMA Project 20201005215.

\section{REFERENCES}

[1] S. Evje and K.H. Karlsen, Monotone difference approximations of $B V$ solutions to degenerate convection-diffusion equations, SIAM J Numer Anal 37 (2000), 1838-1860.

[2] B. Engquist and S. Osher, One-sided difference approximations for nonlinear conservation laws, Math Comp 36 (1981), $321-351$.

[3] C.D. Acosta and C.E. Mejía, Stabilization of explicit methods for convection-diffusion problems by discrete mollification, Comput Math Applic 55 (2008), 363-380.

[4] C.D. Acosta and C.E. Mejía, Approximate solution of hyperbolic conservation laws by discrete mollification, Appl Numer Math 59 (2009), 2256-2265.

[5] D.A. Murio, The Mollification Method and the Numerical Solution of Ill-Posed Problems, John Wiley, New York, 1993.

[6] D.A. Murio, Mollification and space marching, in: K. Woodbury (Ed.), Inverse Engineering Handbook, CRC Press, Boca Raton, 2002. 
[7] C.E. Mejía and D.A. Murio, Numerical identification of diffusivity coefficient and initial condition by discrete mollification, Comput Math Applic 30 (1995), 35-50.

[8] C.E. Mejía and D.A. Murio, Numerical solution of generalized IHCP by discrete mollification, Comput Math Applic 32 (1996), 33-50.

[9] D.A. Murio, C.E. Mejía and S. Zhan, Some applications of the mollification method, in: M. Lassonde (Ed.), Approximation, Optimization and Mathematical Economics, Physica-Verlag, 2001, pp. 213-222.

[10] C.D. Acosta and C.E. Mejía, A mollification-based operator splitting method for convection-diffusion equations, Comput Math Applic, to appear.

[11] K.H. Karlsen and N.H. Risebro, An operator splitting method for nonlinear convection-diffusion equations, Numer Math 77 (1997), 365-382.

[12] M.G. Crandall and A. Majda, Monotone difference approximations for scalar conservation laws, Math Comp 34 (1980), $1-21$.

[13] A. Harten, J.M. Hyman and P.D. Lax, On finite difference approximations and entropy conditions for shocks, Comm Pure Appl Math 29 (1976), 297-322.

[14] S. Evje and K.H. Karlsen, Degenerate convection-diffusion equations and implicit monotone difference schemes. In: H. Freistühler and G. Warnecke (eds.), Hyperbolic Problems: Theory, Numerics, Applications, Birkhäuser-Verlag, Basel, Switzerland, 1999, pp. 285-294.

[15] R. Bürger, A. Coronel and M. Sepúlveda, A semi-implicit monotone difference scheme for an initial-boundary value problem of a strongly degenerate parabolic equation modelling sedimentation-consolidation processes, Math Comp 75 (2006), 91112.

[16] K.H. Karlsen and N.H. Risebro, Convergence of finite difference schemes for viscous and inviscid conservation laws with rough coefficients, M2AN Math Model Numer Anal 35 (2001), 239-269.

[17] K.H. Karlsen, N.H. Risebro and J.D. Towers, Upwind difference approximations for degenerate parabolic convectiondiffusion equations with a discontinuous coefficient, IMA J Numer Anal 22 (2002), 623-664.

[18] K.H. Karlsen, N.H. Risebro and J.D. Towers, $L^{1}$ stability for entropy solutions of nonlinear degenerate parabolic convectiondiffusion equations with discontinuous coefficients, Skr K Nor Vid Selsk 3 (2003), 49 pp.

[19] R. Bürger, K.H. Karlsen and J.D. Towers, A mathematical model of continuous sedimentation of flocculated suspensions in clarifier-thickener units, SIAM J Appl Math 65 (2005), 882-940.

[20] A. Coronel, F. James and M. Sepúlveda, Numerical identification of parameters for a model of sedimentation processes, Inverse Problems 19 (2003), 951-972.

[21] K.H. Karlsen and N.H. Risebro, On the uniqueness and stability of entropy solutions of nonlinear degenerate parabolic equations with rough coefficients, Discr Cont Dyn Syst 9 (2003), 1081-1104.

[22] L.C. Evans and R.C. Gariepy, Measure Theory and Fine Properties of Functions. CRC Press, Boca Raton, FL, 1992.

[23] H. Holden, K.H. Karlsen and K.-A. Lie, Operator splitting methods for degenerate convection-diffusion equations II: numerical examples with emphasis on reservoir simulation and sedimentation, Comput Geosci 4 (2000), 287-322.

[24] R. Bürger and K.H. Karlsen, On a diffusively corrected kinematic-wave traffic model with changing road surface conditions, Math Models Methods Appl Sci 13 (2003), 1767-1799.

[25] S. Berres, R. Bürger, K.H. Karlsen and E.M. Tory, Strongly degenerate parabolic-hyperbolic systems modeling polydisperse sedimentation with compression, SIAM J Appl Math 64 (2003), 41-80.

[26] R. Bürger and K.H. Karlsen, On some upwind schemes for the phenomenological sedimentation-consolidation model, J Eng Math 41 (2001), 145-166.

[27] R. Bürger, A. Kozakevicius and M. Sepúlveda, Multiresolution schemes for strongly degenerate parabolic equations in one space dimension, Numer Meth Partial Diff Eqns 23 (2007), 706-730. 